\title{
Richness of compact radio sources in NGC 6334D to F
}

\author{
S.-N. X. Medina ${ }^{1}$, S. A. Dzib ${ }^{1}$, M. Tapia ${ }^{2}$, L. F. Rodríguez ${ }^{3}$, and L. Loinard ${ }^{3,4}$ \\ 1 Max-Planck-Institut für Radioastronomie, Auf dem Hügel 69, 53121 Bonn, Germany \\ e-mail: smedina@mpifr-bonn.mpg.de \\ 2 Instituto de Astronomía, Universidad Nacional Autónoma de México, Ensenada, B. C., CP 22830, Mexico \\ 3 Instituto de Radioastronomía y Astrofísica, Universidad Nacional Autónoma de México, 58089 Morelia, Mexico \\ ${ }^{4}$ Instituto de Astronomía, Universidad Nacional Autónoma de México, Apartado Postal 70-264, CdMx CP 04510, Mexico \\ Received 14 August 2017 / Accepted 3 November 2017
}

\begin{abstract}
Context. The presence and properties of compact radio sources embedded in massive star forming regions can reveal important physical properties about these regions and the processes occurring within them. The NGC 6334 complex, a massive star forming region, has been studied extensively. Nevertheless, none of these studies has focused in its content in compact radio sources.

Aims. Our goal here is to report on a systematic census of the compact radio sources toward NGC 6334, and their characteristics. This will be used to attempt to define their very nature.

Methods. We used the VLA C band (4-8 GHz) archive data with $0^{\prime \prime} .36$ (500 AU) of spatial resolution and noise level of $50 \mu \mathrm{Jy} \mathrm{bm}^{-1}$ to carry out a systematic search for compact radio sources within NGC 6334. We also searched for infrared counterparts to provide some constraints on the nature of the detected radio sources.

Results. A total of 83 compact sources and three slightly resolved sources were detected. Most of them are here reported for the first time. We found that 29 of these 86 sources have infrared counterparts and three are highly variable. Region D contains 18 of these sources. The compact source toward the center, in projection, of region $\mathrm{E}$ is also detected.

Conclusions. From statistical analyses, we suggest that the 83 reported compact sources are real and most of them are related to NGC 6334 itself. A stellar nature for 27 of them is confirmed by their IR emission. Compared with Orion, region D suffers a deficit of compact radio sources. The infrared nebulosities around two of the slightly resolved sources are suggested to be warm dust, and we argue that the associated radio sources trace free-free emission from ionized material. We confirm the thermal radio emission of the compact source in region E. However, its detection at infrared wavelengths implies that it is located in the foreground of the molecular cloud. Finally, three strongly variable sources are suggested to be magnetically active young stars.
\end{abstract}

Key words. radio continuum: stars - stars: formation - radiation mechanisms: thermal - radiation mechanisms: non-thermal techniques: interferometric

\section{Introduction}

Massive star forming regions contain high- and low-mass stars, which often present radio emission from different origins. The high-mass stars may ionize the medium around them, creating an $\mathrm{H}_{\text {II }}$ region, which produces free-free emission detectable at radio wavelengths. Depending on the size and density of the ionized region they can be classified as classic, compact (C), ultra-compact (UC), and hyper-compact (HC) $\mathrm{H}_{\text {II }}$ regions (Kurtz 2005). Also, massive stars may produce radio emission from their ionized winds (Contreras et al. 1996) and, in massive multiple stellar systems, from their wind collision regions (e.g., Ortiz-León et al. 2011). On the other hand, young lowmass stars may produce free-free radio emission in their jets (Anglada 1996), from their externally ionized disk by the UV photons of an OB star (proplyds; O'dell et al. 1993), and gyrosynchrotron radio emission when they are magnetically active. Even for nearby star forming regions $(d<2 \mathrm{kpc})$, the radio emission of $\mathrm{HC} \mathrm{H}_{\text {II }}$ regions and from low-mass stars occurs on subarcsecond scales (see Rodríguez et al. 2012, for a detailed description on the characteristics of the different types of compact radio emission). Massive star forming regions contain many unresolved, or slightly resolved, radio sources (e.g. Orion and M17; Forbrich et al. 2016; Rodríguez et al. 2012). The compact radio sources have long been known to exist also within extended HII regions such as Orion, and have recently been found to be abundant in other cases. They are important because they might play a role in the time evolution of the HII regions themselves. In this work we present the first radio detection of a large number of radio sources in NGC 6334.

The giant molecular cloud NGC 6334 is a complex with very active spots of massive star formation at different evolutionary stages (see Persi \& Tapia 2008, for a detailed review). Located at the relative nearby distance of $1.34_{-0.12}^{+0.15} \mathrm{kpc}$ (Reid et al. 2014) it is an ideal target for the study of the different phases of massive star formation and the detailed analysis of its content in compact radio sources may provide strong clues on the phases to which they belong.

Early radio maps with high resolution $\left(\sim 1^{\prime \prime}\right)$ of NGC 6334 showed the existence of six strong radio sources which were named as sources NGC 6334A to F (Rodríguez et al. 1982, from now on we will refer to them only by their reference letter). Source B is extragalactic (Moran et al. 1990; Bassani et al. 2005), while the others are compact and UC-H II regions. In this manuscript we will focus on the study of compact sources around the regions $\mathrm{D}, \mathrm{E}$, and $\mathrm{F}$ and their surroundings, including the region called NGC 6334I(N) (hereafter I(N)). These sources are located in the north-east portion of the cloud. 
Briefly described, source $\mathrm{D}$ is an evolved $\mathrm{H}_{\text {II }}$ region with a nearly circular shape of approximate radius of $75^{\prime \prime}(0.5 \mathrm{pc})$, centered on what appears to be one of its main ionizing early B type stars, 2MASS J17204800-3549191, reddened by about $A_{V}=10$ (Straw et al. 1989). At its western edge, the expansion of the $\mathrm{H}_{\text {II }}$ region seems to be halted by a dense dark cloud, where CXOU 172031.76-355111.4 (also known as 2MASSJ172044663549168 or NGC 6334II-23), a more luminous late O-type ionizing star, lies (Feigelson et al. 2009; Straw \& Hyland 1989). This interaction appears to have triggered a second star formation stage in the region inside the dense dark cloud (Persi \& Tapia 2008). Regions E and F, widely studied at several wavelengths, are $\mathrm{H}_{\text {II }}$ regions that contain many signs of star formation (Persi \& Tapia 2008 and references therein). The $\mathrm{C}-\mathrm{H}_{\text {II }}$ region $\mathrm{E}$ shows an extended shell-like structure with a compact radio source at its center (Carral et al. 2002). Source F is a younger and complex UC $\mathrm{H}_{\text {II }}$ region with a cometary shape and a radio flux of $\sim 3 \mathrm{Jy}$. This source and its surroundings are collectively known as NGC 6334I. Finally, the region I(N) is the youngest of all, as it has just started to form massive stars (Rodríguez et al. 2007; Hunter et al. 2006).

\section{Observations}

We have used an archival observation of NGC 6334 obtained with the Karl G. Jansky Very Large Array (VLA) telescope of the $\mathrm{NRAO}^{1}$ in the $C$-band ( 4 to $8 \mathrm{GHz}$ ) obtained as part of the project $10 \mathrm{C}-186$. The data were taken on 7 July 2011, while the array was in A-configuration, its most extended. Two sub-bands, each $1 \mathrm{GHz}$ wide and centered at 5.0 and $7.1 \mathrm{GHz}$, respectively, were recorded simultaneously. The average frequency of the whole observation is $6.0 \mathrm{GHz}$. The quasars J1717-3342, J19242914 and J1331+3030, were used as the gain, bandpass, and flux calibrators, respectively. The total time spent on source was 84 minutes. First results of this observation were reported by Hunter et al. (2014) and Brogan et al. (2016), we refer the reader to these papers for further details of the observation.

The data were edited, calibrated and imaged using the software CASA with the help of the VLA Calibration Pipeline. The image at $6.0 \mathrm{GHz}$ was produced by combining the two subbands using a multi-frequency deconvolution software (e.g., Rau $\&$ Cornwell 2011) and with a pixel size of $0{ }^{\prime \prime} .06$. The resulting beam size is $0^{\prime \prime} .67 \times 00^{\prime \prime} .19$; PA $=-2.7$. The used weighting scheme was intermediate between natural and uniform (robust = 0, Briggs 1995). Similar images were also produced for both sub-bands separately, with pixel sizes of $00^{\prime \prime} .06$ and $00^{\prime \prime} 04$ for 5.0 and $7.1 \mathrm{GHz}$, respectively. The beam size for the $5.0 \mathrm{GHz}$ image is $0 \prime \prime 91 \times 0, .25 ; \mathrm{PA}=-2.3$, and $0^{\prime \prime} .66 \times 0, .17$; PA $=-2.8$ for the image at $7.1 \mathrm{GHz}$. All images are corrected for the primary beam response.

\section{Source extraction}

The source extraction was performed using the BLOBCAT software (Hales et al. 2012). This software is a flood fill algorithm that cataloged islands of agglomerated pixels (blobs) within locally varying noise. It is designed for two-dimensional input FITS images of surface brightness (SB). BLOBCAT makes improvements in the morphological assumptions and applies bias corrections to extract blob properties (see Hales et al. 2012, for

\footnotetext{
1 The National Radio Astronomy Observatory is operated by Associated Universities Inc. under cooperative agreement with the National Science Foundation.
}

details). To run BLOBCAT, we also used the rms estimator algorithm implemented within the SExtractor package (Bertin \& Arnouts 1996; Holwerda 2005) to make a suitable noise map of the SB image. We used a mesh size of $60 \times 60$ pixels and signal to noise ratio $(S / N)=5$ as threshold for detecting blobs for the $6.0 \mathrm{GHz}$ image. For the 5.0 and $7.1 \mathrm{GHz}$ images the mesh size was $80 \times 80$ pixels and $S / N=3$ as a threshold, but in this case only sources that were also founded in the $6.0 \mathrm{GHz}$ map were considered as real. The previous calculations were made following the derivations of Hales et al. (2012).

The expected number of false detections is calculated using the complementary cumulative distribution function $\Phi(x)=$ $1-\phi(x)$, where $\phi(x)$ is the cumulative distribution function. Assuming that the noise in our radio maps follows a Gaussian distribution

$\phi(x)=\frac{1}{2}\left[1+\operatorname{erf}\left(\frac{x}{\sqrt{2}}\right)\right]$

where erf is the error function given by

$\operatorname{erf}(x)=\frac{1}{\sqrt{\pi}} \int_{-x}^{x} \mathrm{e}^{-t^{2}} \mathrm{~d} t$

$\Phi(x)$ is the probability that a value of a standard normal random variable $\mathrm{X}$ will exceed an $x$ level. So, the probability that any independent pixel (synthesized beam) will have a value up to $5 \sigma$ is $\Phi(5) \approx 3 \times 10^{-7}$. In consequence, we expect a total of one source above $5 \sigma$ in our $6 \mathrm{GHz}$ maps, and conclude that essentially all our sources cataloged in the region are real. In consequence, the sources above $5 \sigma$ at $6.0 \mathrm{GHz}$ are trustworthy. Even so, we carried out a visual inspection of all these sources to confirm their detection. We excluded the artifacts located close to the edge of the image.

\section{Results}

\subsection{Radio sources}

We obtain an image of $10^{\prime} \times 10^{\prime}$, which is displayed in Fig. 1, combining the two observed sub-bands. The noise level is position dependent and produces the following two effects. The first is that the noise increases at the edges of the image, which is expected from the Gaussian primary beam pattern. The second is due to the imperfect sampling of the UV-space and it mainly affects areas around the extended strong emission. Thus, the noise level is close to $50 \mu \mathrm{Jy}$ near the $\mathrm{H}_{\text {II }}$ regions, but only about $8 \mu \mathrm{Jy}$ in areas free of extended emission and not far from the center of the image. A similar effect is present in the Orion observations of Forbrich et al. (2016).

In Fig. 1, the $\mathrm{UC} \mathrm{H}_{\text {II }}$ region $\mathrm{F}$ and $\mathrm{C} \mathrm{H}_{\text {II }}$ region $\mathrm{E}$ can be immediately appreciated, while only a fraction of the extended emission of source D is recovered. Only a small portion of region $\mathrm{C}$ falls inside the primary beam of this observation. In addition to the extended emission of the $\mathrm{H}_{\text {II }}$ regions, a total of 83 compact $^{2}$ radio sources were also detected (Table A.1), including those previously reported in the $\mathrm{E}, \mathrm{I}$, and $\mathrm{I}(\mathrm{N})$ region. The compact sources cannot be easily appreciated in this large field figure and they are represented by small red circles. The astrometric accuracy for the positions is better than $0^{\prime \prime} .1$, and we have used the positions of the sources to name them according to the IAU suggestion.

\footnotetext{
2 Compact sources are defined here as source whose deconvolved sizes are similar to, or smaller than the synthesized beam.
} 


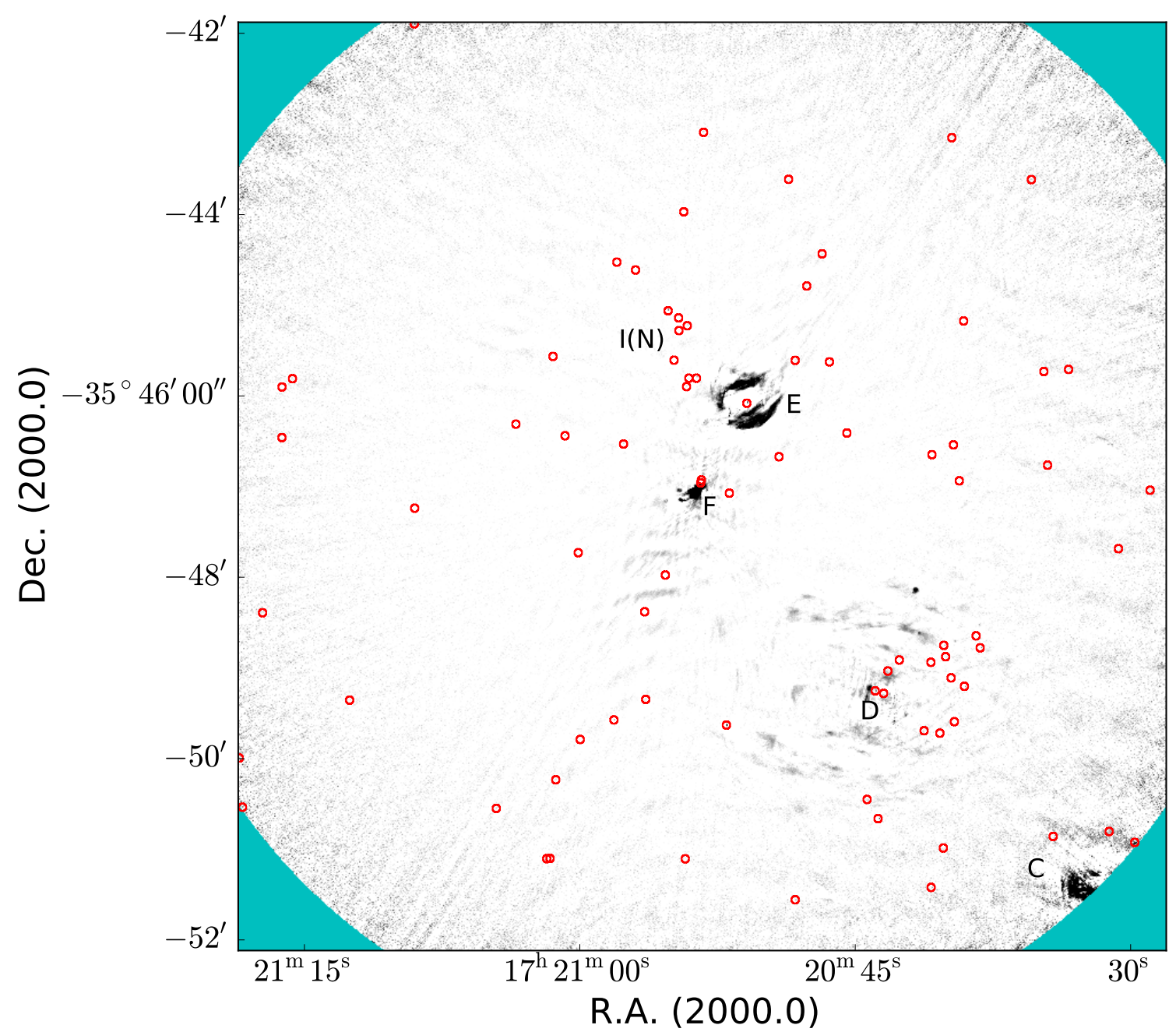

Fig. 1. VLA image of NGC 6334E to F and I(N). Small circles indicate the position of the detected compact sources.

Using Eq. (A.11) from Anglada et al. (1998), the expected number of background extragalactic sources in the imaged area with flux densities above $50 \mu \mathrm{Jy}$ is $7 \pm 3$. This previously computed number only reflects an upper limit because the emission of the extended sources affects the noise distribution and this changes the value of the expected background sources. Most of the detected compact radio sources are Galactic objects and most probably related to the NGC 6334 complex.

Region D, presented in Fig. 2, contains a significant fraction of all of the detected compact radio sources. Also, at the north side of this region there are two cometary shaped radio sources and at its center a double source. All of them, the compact radio sources, the cometary radio sources and the double radio sources, are reported here for the first time.

In order to compute the spectral index $\left(\alpha ; S_{v} \propto v^{\alpha}\right)$, we measured the flux density of the detected sources in the $5.0 \mathrm{GHz}$ and $7.1 \mathrm{GHz}$ maps and calculated the slope between both points. The error was calculated by using the standard error propagation theory. As the field of view of the map at $7.1 \mathrm{GHz}$ is smaller than at $5.0 \mathrm{GHz}$, we could not calculate the spectral index for some sources that lie outside the edges of the $7.1 \mathrm{GHz}$ image. Additionally, some sources were detected in one band but not in the other, in those cases we just calculate a limit for $\alpha$. The spectral index for each individual source is shown in Table A.1.

Using the SIMBAD database we searched for counterparts of detected radio sources. Inside a radius of 0".5 from the VLA position, we found 17 radio sources with counterparts at different wavelengths and are shown in Table A.1. Additionally, the source SSTU J172053.96-3545.6 is at an angular distance of 0".9 from VLA J172054.06-354548.4. This shift is larger than the combined position errors of both telescopes (pointing accuracy for IRAC is $\sim 0^{\prime \prime} 5$ ) and indicates that these sources are not the same object, but we cannot discard the possibility that they may be related (e.g., a binary system). Finally, source [S2000e] SM6 is at an angular distance from source VLA J172053.26-354305.7 of $\sim 1^{\prime \prime} 2$, this agrees within the position error of $2^{\prime \prime}$ for the millimeter source (Sandell 2000). To look for more infrared counterparts we performed a more detailed search, described in the next section.

\subsection{Infrared counterparts}

We searched for infrared counterparts of the compact radio sources searched in Sect. 4.1. We use the source catalog from the Spitzer space mission as well as published near- and mid-IR photometry data by Willis et al. (2013) and Tapia et al. (1996). We complemented the search with unpublished $J H K_{\mathrm{s}}$ photometric data from M. Tapia (in prep.). Defining reliable criteria for assigning a positive infrared counterpart to a compact radio source is not straightforward, as we are dealing with several infrared sets of infrared photometric measurements, namely from Spitzer images in the mid-IR and from ground-based observations with 


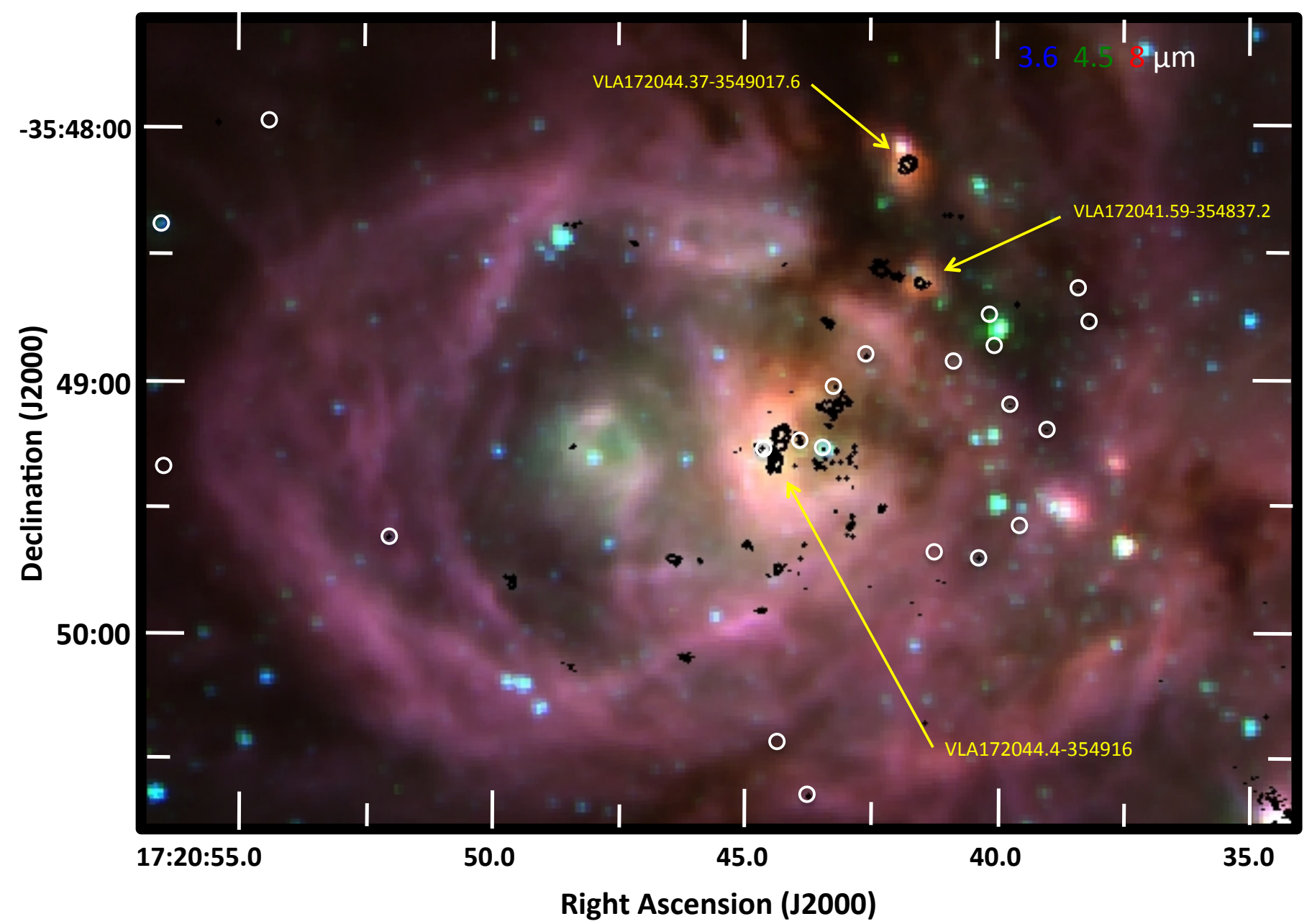

Fig. 2. Composite Spitzer/GLIMPSE infrared image of NGC 6334D. Small circles indicate the position of the detected compact radio sources. Contours trace the radio continuum image at $6.0 \mathrm{GHz}$, and the contour levels are at 90, 180, 300, 450, 750, and $1500 \mu \mathrm{Jy} / \mathrm{beam}$. Slightly extended radio sources are indicated.

three different telescopes (and set-ups) in the near-IR. For the present work, we cross-checked the coordinates of the VLA compact sources with those from the Spitzer and also from the ground-based images. We selected those with coordinates coinciding (in all wavelength ranges) within 0 '.9 or less to obtain a list of candidate counterparts. We then examined by eye each source on all available individual images (i.e., each wavelength) and checked for the consistency of the corresponding flux measurements. The mean differences in the radio, mid- and near-IR source positions for the 27 counterparts were -0 ".07 in right ascension and $+0^{\prime \prime} .07$ in declination, with standard deviations of $0 \prime$ !44 and $0 \prime$ !30, respectively. In all, no systematic coordinate offsets were found in the (small) area covered by this survey.

From the 83 VLA sources in Table A.1, only 27 IR counterparts were found. Interestingly, ten of these infrared sources are inside the dark cloud in region D. The ground-based nearIR and Spitzer/IRAC mid-IR photometry of all these sources are listed in Table 1. The diagnostic two-color and color-magnitude diagrams are presented in Figs. 3-6. The $J-H$ vs. $H-K_{\mathrm{s}}$ and the $H-K_{\mathrm{s}}$ vs. $K_{\mathrm{s}}-$ [3.6] diagrams are accurate tools for discriminating reddened photospheres (those lying along the reddening vectors) from stars with disks (i.e., near-IR excesses). The latter stars would lie shifted towards redder colors in these diagrams. The amount of dust extinction (i.e., the value of $A_{V}$ along the line of sight) for the former set of stars, is determined by the colorexcess indices, under a "standard" reddening law, represented by the reddening vectors. References for the intrinsic colors and reddening law assumed for determining these parameters are given in the figure captions.

The color-magnitude plot $K_{\mathrm{s}}$ vs. $H-K_{\mathrm{s}}$, on the other hand, is the best tool for estimating approximate spectral types and intra-cloud extinction values for embedded stellar sources located at the distance of the star formation complex $(d=1.34$ $\mathrm{kpc}$ and foreground value of $A_{V}=1.0$ in this case). Finally, the IRAC two-color magnitude [3.6]-[4.5] vs. [4.5]-[5.8] diagram provides a simple diagnostics for classifying the evolutionary status of YSOs and of emission-line-dominated regions (mainly Polycyclic Aromatic Hydrocarbons, PAHs, or shocked molecular hydrogen). This is described in detail by Ybarra et al. (2014) and references therein. The combined results for these analyses in terms of the derived properties of a number of individual sources are listed in the last column of Table 1, where we also indicate whether it was detected as an X-ray source (within 0".6) by Feigelson et al. (2009).

\section{Discussion}

To define the nature of the radio emission of the compact sources, we need information on the different characteristics ( $\alpha$, polarization, and time variability). However we are able to achieve only a rough measure of the spectral index, which only gives clues to the emission mechanism. While thermal free-free 
Table 1. Infrared photometry for stellar counterparts of compact radio sources.

\begin{tabular}{|c|c|c|c|c|c|c|c|c|}
\hline ID & $J$ & $H$ & $K$ & [3.6] & [4.5] & {$[5.8]$} & [8] & Notes \\
\hline \multicolumn{9}{|c|}{ Sources inside region D } \\
\hline 3 & - & $17.76 \pm 0.04$ & $15.25 \pm 0.02$ & $12.33 \pm 0.18$ & $12.41 \pm 0.19$ & - & - & $2,4,9, X$ \\
\hline 4 & $16.75 \pm 0.02$ & $15.30 \pm 0.08$ & $13.89 \pm 0.07$ & - & - & - & - & $2,4,8, X$ \\
\hline 5 & $\ldots$ & - & 15.980 .10 & $12.65 \pm 0.10$ & $11.98 \pm 0.11$ & - & - & \\
\hline 8 & $19.72 \pm 0.11$ & $15.47 \pm 0.02$ & $13.15 \pm 0.02$ & $11.61 \pm 0.16$ & $11.32 \pm 0.15$ & - & - & $1,8, \mathrm{X}$ \\
\hline 9 & - & - & $15.55 \pm 0.05$ & - & - & $9.78 \pm 0.12$ & $7.25 \pm 0.13$ & $\mathrm{X}$ \\
\hline 10 & - & $17.86 \pm 0.12$ & $16.24 \pm 0.08$ & - & - & - & - & \\
\hline 11 & $15.15 \pm 0.02$ & $13.24 \pm 0.02$ & $12.36 \pm 0.03$ & - & - & - & - & $2,4,8, \mathrm{X}$ \\
\hline 12 & - & $17.44 \pm 0.12$ & $14.27 \pm 0.05$ & $12.47 \pm 0.18$ & - & - & - & $1,9, \mathrm{X}$ \\
\hline 13 & $16.36 \pm 0.10$ & $12.34 \pm 0.08$ & $10.14 \pm 0.09$ & $8.53 \pm 0.04$ & $7.87 \pm 0.05$ & $7.52 \pm 0.04$ & $6.85 \pm 0.16$ & $1,9,6, \mathrm{X}$ \\
\hline 14 & $17.48 \pm 0.08$ & $15.56 \pm 0.04$ & $13.58 \pm 0.03$ & $11.37 \pm 0.10$ & $10.21 \pm 0.10$ & - & - & $2,9,4,5$ \\
\hline 15 & $11.34 \pm 0.03$ & $9.79 \pm 0.03$ & $8.93 \pm 0.03$ & $8.06 \pm 0.12$ & $7.54 \pm 0.11$ & $6.78 \pm 0.07$ & $99.99 \pm 9.99$ & $1,4,5,6, X$ \\
\hline \multicolumn{9}{|c|}{ Remaining sources } \\
\hline 25 & $11.93 \pm 0.02$ & $9.97 \pm 0.03$ & $9.18 \pm 0.02$ & $8.12 \pm 0.03$ & $7.84 \pm 0.01$ & $7.37 \pm 0.03$ & $6.77 \pm 0.06$ & $1,8,4$ \\
\hline 27 & $16.49 \pm 0.01$ & $14.18 \pm 0.02$ & $13.00 \pm 0.01$ & $11.96 \pm 0.05$ & $12.13 \pm 0.05$ & $10.61 \pm 0.09$ & $8.73 \pm 0.13$ & $2,4, X$ \\
\hline 35 & - & - & $16.60 \pm 9.15$ & $13.14 \pm 0.03$ & $12.00 \pm 0.03$ & - & - & 5 \\
\hline 38 & $14.28 \pm 0.02$ & $12.78 \pm 0.01$ & $11.95 \pm 0.01$ & $11.05 \pm 0.07$ & $10.50 \pm 0.10$ & - & - & 2,8 \\
\hline 39 & - & - & - & $14.07 \pm 0.03$ & $10.75 \pm 0.03$ & $8.89 \pm 0.02$ & $7.68 \pm 0.03$ & 5,7 \\
\hline 42 & - & - & $14.40 \pm 0.02$ & $9.49 \pm 0.03$ & $7.29 \pm 0.07$ & $5.82 \pm 0.06$ & $4.46 \pm 0.07$ & $5,7,9$, CHII-E \\
\hline 43 & - & $16.48 \pm 0.04$ & $14.09 \pm 0.04$ & 9.250 .08 & $7.27 \pm 0.06$ & $6.08 \pm 0.04$ & $5.08 \pm 0.05$ & $4,5,7$ \\
\hline 44 & $15.98 \pm 0.01$ & $13.53 \pm 0.01$ & $12.40 \pm 0.02$ & $10.93 \pm 0.07$ & $11.06 \pm 0.05$ & $10.09 \pm 0.08$ & - & $2,8,5, X$ \\
\hline 45 & - & - & - & $13.22 \pm 0.08$ & $12.02 \pm 0.03$ & $10.99 \pm 0.08$ & - & $5,7, X$ \\
\hline 49 & - & - & - & $13.28 \pm 0.03$ & $12.28 \pm 0.09$ & - & - & $\mathrm{X}$ \\
\hline 51 & $16.95 \pm 0.02$ & $13.83 \pm 0.03$ & $12.20 \pm 0.03$ & $10.59 \pm 0.03$ & $10.05 \pm 0.04$ & $10.88 \pm 0.19$ & $8.65 \pm 0.04$ & $1,4,5,6,8, X$ \\
\hline 52 & - & $17.49 \pm 0.04$ & $15.88 \pm 0.05$ & - & - & - & - & \\
\hline 53 & - & - & 16.090 .05 & $11.80 \pm 0.02$ & $10.17 \pm 0.02$ & $9.29 \pm 0.02$ & $8.67 \pm 0.02$ & $5,7, X$ \\
\hline 60 & $10.97 \pm 0.03$ & $10.45 \pm 0.03$ & $10.10 \pm 0.02$ & $9.74 \pm 0.07$ & $9.68 \pm 0.06$ & $9.46 \pm 0.07$ & - & 3 \\
\hline 65 & - & - & - & $15.59 \pm 0.03$ & $12.17 \pm 0.07$ & $12.01 \pm 0.02$ & $9.12 \pm 0.15$ & 5 \\
\hline 75 & $19.40 \pm 0.07$ & $16.21 \pm 0.02$ & $14.67 \pm 0.02$ & $13.58 \pm 0.02$ & $13.44 \pm 0.02$ & - & - & 3,8 \\
\hline
\end{tabular}

Notes. Units of the IR values are magnitudes. $1=$ early B spectral type, $2=$ late B to early A spectral type, $3=$ late-type, $4=$ near-IR excess, $5=$ mid-IR excess, $6=$ Class II, $7=$ Class I, $8=10<A_{V}<28,9=A_{V}>28$, and X $=$ X-ray source (Feigelson et al. 2009).

radio emission has spectral indices with values $-0.1 \leq \alpha \leq+2.0$, gyrosynchrotron emission has values in the range $-2.0 \leq \alpha \leq$ +2.0 , and optically-thin synchrotron emission from wind collision regions has values $\alpha \sim-0.7$ (see Rodríguez et al. 2012, and references therein).

\subsection{Region $D$}

\subsubsection{Compact sources}

Although the reported compact radio sources are spread over the entire observed area, there is a group of 15 objects, reported here for the first time, that is well concentrated (within a radius of $0.3 \mathrm{pc}$ ) in the dark cloud on the western edge of region D. Of these, $73 \%$ (11 sources) have unresolved infrared counterparts (see Fig. 2) and all but three are X-ray emitters
(Feigelson et al. 2009). In the first parts of Tables A.1 and 1 we list the observational data of the compact sources in region D that we now focus on.

The clustering of compact radio sources clearly coincides with the densest part of the dark cloud, where one would suspect to have the latest stage of star formation in the region. From the near-IR colors, we can distinguish only four stars that have a late-O or early-B spectral types, while the rest are less massive. Two of these three massive stars have a negative spectral index, and thus their radio emission may originate in wind collision regions. The majority of the remaining sources (eight sources) have negative spectral indices (non-thermal) values and only three are positive. The association of these sources with $\mathrm{X}$-ray emission is compatible with a non-thermal origin for the radio emission, but there are a few cases in which the spectral index is positive in spite of the presence of an X-ray source 
Table 2. Infrared photometry around the region of cometary nebulae.

\begin{tabular}{|c|c|c|c|c|c|c|c|c|c|c|}
\hline $\begin{array}{c}\text { RA } \\
(\mathrm{J} 2000)\end{array}$ & $\begin{array}{c}\text { Dec } \\
(\mathrm{J} 2000)\end{array}$ & $J$ & $H$ & $K$ & [3.6] & [4.5] & [5.8] & [8] & Notes* & Other \\
\hline \multicolumn{11}{|c|}{ Around VLA J172041.75-354808.2 } \\
\hline 17:20:41.92 & $-35: 48: 04.7$ & $14.69 \pm 0.01$ & $13.40 \pm 0.01$ & $12.37 \pm 0.02$ & $9.50 \pm 0.15$ & $9.14 \pm 0.12$ & $6.46 \pm 0.12$ & $5.33 \pm 0.12$ & $2,8,4,5,7$ & Mir-1 \\
\hline 17:20:41.93 & $-35: 48: 09.7$ & - & - & - & $10.52 \pm 0.18$ & $9.39 \pm 0.13$ & $6.64 \pm 0.13$ & $5.35 \pm 0.12$ & $5,7, X$ & Mir-2 \\
\hline 17:20:42.31 & $-35: 48: 18.3$ & $21.35 \pm 0.13$ & $17.67 \pm 0.01$ & $14.40 \pm 0.02$ & $10.63 \pm 0.17$ & $9.52 \pm 0.12$ & $8.21 \pm 0.20$ & - & $1,9,4,5,7$ & Mir-3 \\
\hline 17:20:42.19 & $-35: 48: 12.5$ & $19.95 \pm 0.09$ & $17.12 \pm 0.03$ & $15.22 \pm 0.01$ & - & - & - & - & $2,9,4$ & - \\
\hline 17:20:42.00 & $-35: 48: 07.7$ & $18.78 \pm 0.12$ & $16.45 \pm 0.04$ & $15.73 \pm 0.03$ & - & - & - & - & 3 & - \\
\hline 17:20:42.02 & $-35: 48: 11.0$ & - & $17.95 \pm 0.05$ & $15.80 \pm 0.09$ & - & - & - & - & 2,9 & - \\
\hline 17:20:42.49 & $-35: 48: 09.5$ & $21.48 \pm 0.12$ & $19.49 \pm 0.24$ & $16.56 \pm 0.04$ & - & - & - & - & 2,4 & - \\
\hline 17:20:42.49 & $-35: 48: 08.5$ & - & - & $17.2 \pm 0.2$ & - & - & - & - & - & \\
\hline 17:20:42.48 & $-35: 48: 03.8$ & - & - & $16.9 \pm 0.2$ & - & - & - & - & - & - \\
\hline $17: 20: 42.29$ & $-35: 48: 03.2$ & $20.01 \pm 0.10$ & $17.93 \pm 0.11$ & $16.79 \pm 0.04$ & - & - & - & - & 3 & - \\
\hline $17: 20: 42.20$ & $-35: 48: 02.6$ & $20.33 \pm 0.17$ & $17.49 \pm 0.11$ & $16.52 \pm 0.06$ & - & - & - & - & 3 & - \\
\hline 17:20:42.02 & $-35: 49: 10.1$ & - & $17.42 \pm 0.03$ & $16.70 \pm 0.07$ & - & - & - & - & - & - \\
\hline 17:20:42.15 & $-35: 48: 17.8$ & - & - & $17.0 \pm 0.2$ & - & - & - & - & - & - \\
\hline 17:20:42.11 & $-35: 48: 13.2$ & - & - & $16.05 \pm 0.10$ & - & - & - & - & - & - \\
\hline 17:20:42.10 & $-35: 48: 05.2$ & - & - & $15.8 \pm 0.2$ & - & - & - & - & - & - \\
\hline
\end{tabular}

Around VLA J172041.59-354837.3

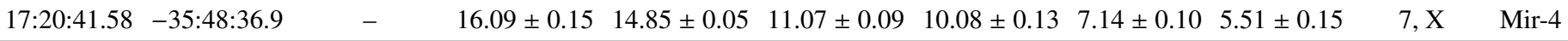

Notes. Units of the IR values are magnitudes. ${ }^{(*)}$ Same as Table 1.

(Dzib et al. 2013). As the sources are compact and likely to be associated with the star forming region, an attractive interpretation is that they are magnetically active low mass YSOs with gyrosynchrotron radio emission. However, the errors are large and this will have to be confirmed with multi-epoch monitoring to measure their flux variability, as nonthermal low mass stars tend to be very variable on scales of days (André 1996). These future observations will also help us to obtain a better estimate of their spectral indices.

It is worth highlighting that in the HII region associated with the Orion core (at $d \simeq 400$ pc; Menten et al. 2007; Kounkel et al. 2017), there is a significant population of YSOs that produce radio emission (e.g., Forbrich et al. 2016; Zapata et al. 2004). In comparison with region $\mathrm{D}$, if the Orion core were located at $1.34 \mathrm{kpc}$, the distance of the NGC 6334 complex, the flux density of the 556 compact sources detected in Forbrich at al. (2016) would be 11.2 times lower. Considering $50 \mu \mathrm{Jy}$ as the detection threshold, only 47 compact sources from Forbrich et al. (2016) could be detected. We detect only 14 compact sources, or about one third of the expectation based on the Orion population. This is consistent with other similar comparisons (e.g., Masqué et al. 2017), which may indicate that the Orion core may be richer in radio sources than other similar regions. Also, our images are highly contaminated by the extended emission making it difficult to identify all compact sources. Finally, there may be more sources below our detection threshold but we need deeper radio observations to clarify this point.

\subsubsection{Cometary nebula north $(\mathrm{CNN})=$ VLA J172041.75-354808.2}

Close to the north-western edge of the expanding HII region $\mathrm{D}$, we discovered an extended radio source with a cometary structure, known as cometary nebula north (CNN). Its flux density at $6.0 \mathrm{GHz}$ is $18.4 \pm 0.1 \mathrm{mJy}$ with a peak flux of $385 \pm$ $12 \mu \mathrm{Jy} \mathrm{bm}{ }^{-1}$. The position of its peak flux is RA $=17^{\mathrm{h}} 20^{\mathrm{m}} 41^{\mathrm{s}} .75$; Dec $=-35^{\circ} 48^{\prime} 08^{\prime \prime} .2$.

This peak of the extended radio emission reported here is at the center of a mid-IR nebulosity with an ovoid shape of size around $15^{\prime \prime}$. It is relatively bright in the $4.5,5.8$ and $8 \mu \mathrm{m}$ Spitzer/IRAC images, as shown in the top panel of Fig. 7. Although their spatial resolution $\left(1^{\prime \prime} .5\right.$ to $\left.2^{\prime \prime} .0\right)$ is much worse than that obtained with the VLA, a similar morphology of the diffuse emission is evident. The mid-IR structure is complicated by the presence of at least three bright, unresolved mid-IR sources (Mir-1, Mir-2, and Mir-3), most likely of protostellar nature embedded in the nebulosity. Figure 7-Bottom shows the same field at $2.2 \mu \mathrm{m}$ as observed in excellent seeing conditions $\left(\sim 0^{\prime \prime} .55\right)$ with the 2.5-m DuPont telescope at Las Campanas Observatory (Tapia et al., in prep.). In addition to the mid-IR sources, there are a dozen near-IR stars detected within the nebula. Comparison with the surrounding field shows that at least $60 \%$ of them must be embedded in it. IRAC and $J H K$ photometry of these sources are reported in Table 2 and their colors are plotted in Figs. 3-6.

The nature of the IR sources in CNN can be deduced from the multi-wavelength photometry. From the nebula-subtracted fluxes of sources Mir-1, Mir-2, and Mir-3, we conclude that they are Class I young stellar objects with spectral types earlier than $\mathrm{A}$ and each with quite different characteristics. The notes in Col. 9 of Table 2 summarize the results. Interestingly, in the cometary nebulae there is another emission peak evident in the radio maps at the three frequencies (see Fig. 8) at the position $\mathrm{RA}=17^{\mathrm{h}} 20^{\mathrm{m}} 41$ s 93 ; Dec. $=-35^{\circ} 48^{\prime} 10^{\prime \prime} .5$. This position is consistent, within errors, with the position of source Mir-2 and we suggest that this peak may be its radio counterpart. 


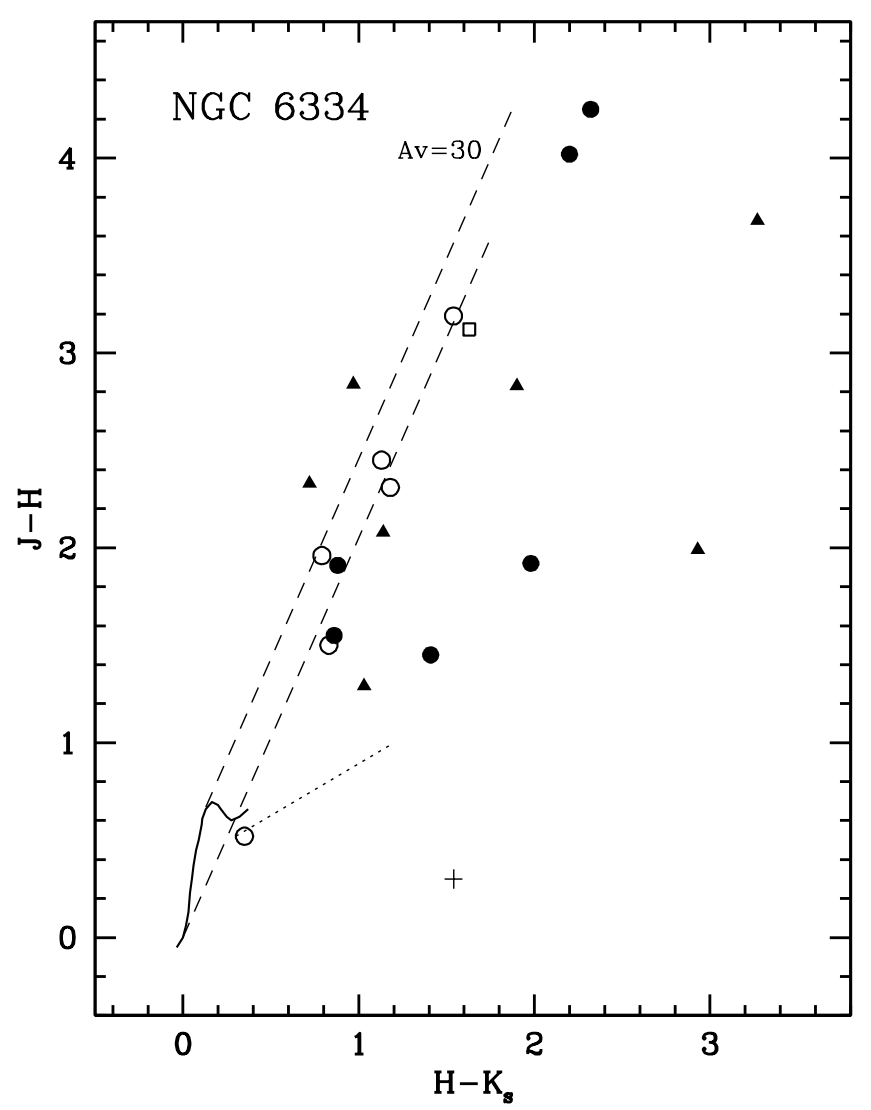

Fig. 3. $J-H$ vs. $H-K_{\mathrm{s}}$ diagram of the near-IR counterparts with measurements in the three bands. Solid circles are sources in the dark cloud west of the HII region D. Solid triangles correspond to sources within the limits of $\mathrm{CNN}$. Open squares are in region $\mathrm{I}(\mathrm{N})$. Open circles are for sources in the rest of the observed field. The solid line marks the locus of the main sequence (Koornneef 1983), the dashed lines delineate the reddening band for all main sequence star and giant stars (Rieke \& Lebofsky 1985). The small plus sign near the bottom of the plot illustrates the maximum photometric errors.

Concerning the diffuse, nebular emission, from the present images, we attempted to measure nebular fluxes at several IR wavelengths. Because of many limitations (poor and very variable resolutions, presence of point-like sources, etc.) we found it impossible to deduce total fluxes reliably. Nevertheless, by integrating the near- and mid-IR flux densities that are well-derived from the ground-based and Spitzer observations and extrapolate them into the far-IR, we can estimate a lower limit to the total IR luminosity of the nebulosity to be $\sim 10^{3} L_{\odot}$. Undoubtedly, this arises from thermal emission of warm dust.

Thus we assume that the $\mathrm{CNN}$ source is an $\mathrm{H}_{\text {II }}$ region, surrounded by a dusty envelope, and derive its properties under that assumption. At a frequency of $6.0 \mathrm{GHz}$, we derive $\mathrm{e}^{3}$ a brightness temperature of $44 \mathrm{~K}$, an electron density of $4.8 \times 10^{3} \mathrm{~cm}^{-3}$, an emission measure of $0.6 \times 10^{6} \mathrm{~cm}^{-6} \mathrm{pc}$, an ionized mass of $8 \times 10^{-3} M_{\odot}$, and a flux of ionizing photons of $1.4 \times 10^{46} \mathrm{~s}^{-1}$ assuming a circular shape with a radius of $2^{\prime \prime}=0.013 \mathrm{pc}$, and an electron temperature of $T_{\mathrm{e}}=10^{4} \mathrm{~K}$. These values are consistent with an UC $\mathrm{H}_{\text {II }}$ region being photoionized by a B0.5 ZAMS star (Kurtz et al. 1994; Panagia 1973). This is consistent with the approximated spectral types of sources Mir-1, Mir-2, and Mir-3.

\footnotetext{
3 We have used the basic equations of brightness temperature, emission measure, electron density, and ionizing photon rate (e.g., Table 4 in Masqué et al. 2017).
}

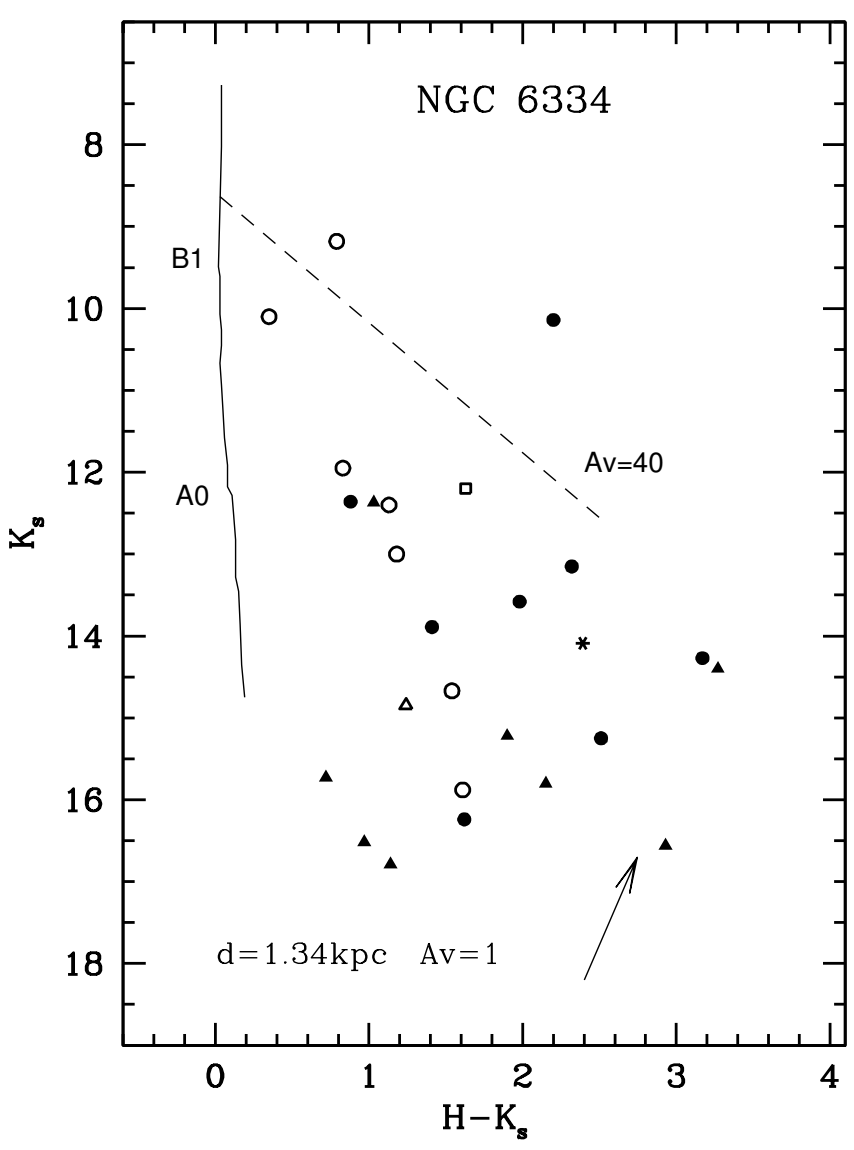

Fig. 4. $K_{\mathrm{s}}$ vs. $H-K_{\mathrm{s}}$ diagram of the near-IR counterparts with measurements in the $H$ and $K_{\mathrm{s}}$ bands. The open triangle refers to Mir-4 in DNS and the rest of the symbols are as in Fig. 3. For reference, the almost vertical solid line delineates the zero-age main sequence (ZAMS; Drilling \& Landolt 2000) for $d=1.34 \mathrm{kpc}$ and $A_{V}=1.0$. The dashed lines are the reddening vectors of length $A_{V}=40$ for B0 ZAMS stars. The arrow represents the average slope of the near-IR emission excess caused by disks around YSOs, as determined by López-Chico \& Salas (2007).

\subsubsection{Cometary Nebula South $(\mathrm{CNS})=$ VLA J172041.59-354837.2}

As can be seen in Fig. 2, a second small mid-IR nebula was found about 27" south of CNN. We refer to this source as cometary nebula south (CNS). Both nebulosities have similar mid- and far-IR colors implying matching dust temperatures, though the integrated Herschel flux at $70 \mu \mathrm{m}$ for CNS is four times fainter than that from CNN. Unfortunately, no photometry could be obtained at longer wavelengths and, thus, no reliable dust temperature can be derived. CNS has at its center a compact near- and mid-IR source ${ }^{4}$ (Mir-4 in Fig. 9 and Table 2). The IR photometry of this source suggests that this is an intermediate luminosity Class I YSO with $A_{V} \simeq 13-16$. This object was also detected in X-rays by Feigelson et al. (2009). At the same position, our $6.0 \mathrm{GHz}$ image shows the presence of a small, roundish radio source with a diameter of around 2 . Its total flux density at $6.0 \mathrm{GHz}$ is $4.5 \pm 0.4 \mathrm{mJy}$. As for $\mathrm{CNN}$, the presence of warm dust also indicates that the radio emission of CNS also traces ionized gas. The derived parameter in this case are a brightness temperature of $39 \mathrm{~K}$, an electron density of $6.2 \times 10^{3} \mathrm{~cm}^{-3}$, an emission

\footnotetext{
4 We note that the neighboring bright star that appears as blue pixels in the mid-IR IRAC image (Fig. 9) is, given its IR colors, a foreground star unrelated to CNS.
} 


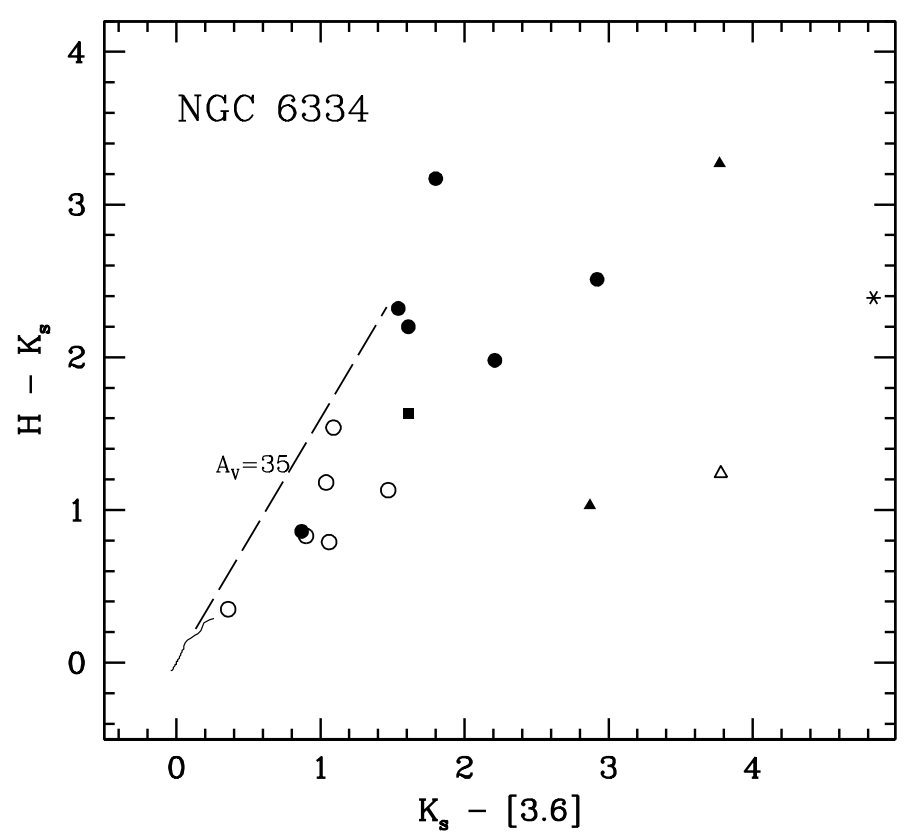

Fig. 5. $H-K_{\mathrm{s}}$ vs. $K_{\mathrm{s}}-[3.6]$ of the IR-counterparts with measurements in the $H, K$, and IRAC channel bands. the solid square corresponds to the central C-HII region E, the asterisk to source \#43 in region F. The rest of the symbols are as in Figs. 3 and 4. The solid line close to the origin represents the locus of the main sequence (Koornneef 1983) and the dashed line represents the reddening vector of length $A_{V}=35$ (Tapia 1981).

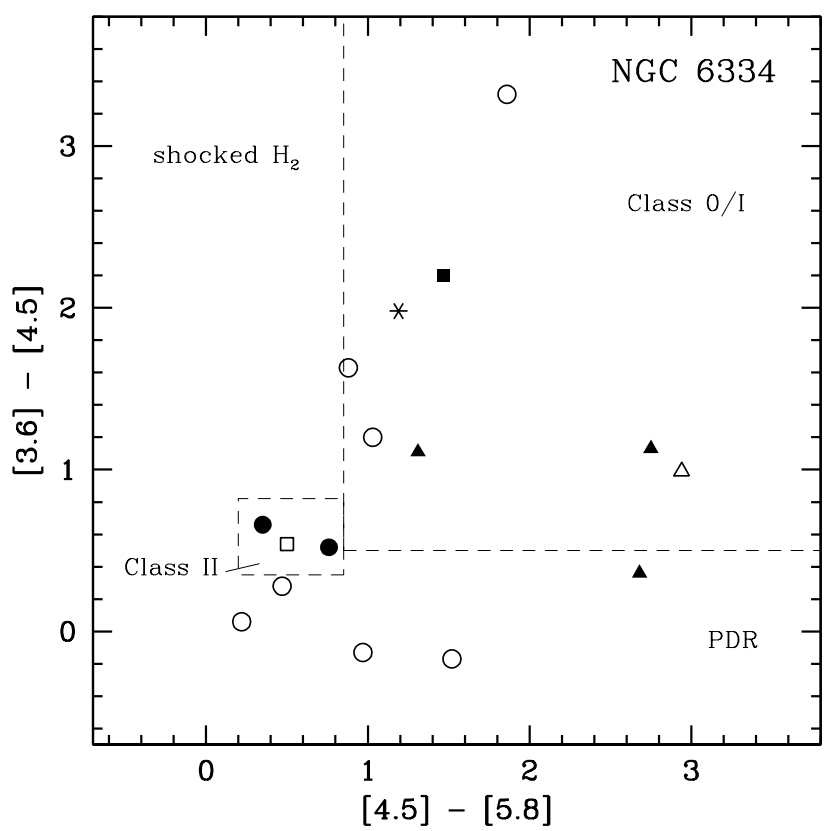

Fig. 6. [3.6] - [4.5] vs. [4.5] - [5.8] diagram of the IR counterparts measured in the three IRAC bands. Symbols are as in Figs. 3 and 5. The dashed rectangles mark the locii of the Class II and Class 0/I sources and the labels "shocked $\mathrm{H}_{2}$ " and "PDR" mark, respectively, the areas occupied by shocked regions emitting molecular hydrogen lines and PAHemission-dominated photodissociation regions (Ybarra et al. 2014).

measure of $0.5 \times 10^{6} \mathrm{~cm}^{-6} \mathrm{pc}$, an ionized mass of $1 \times 10^{-3} M_{\odot}$, and a flux of ionizing photons of $3.0 \times 10^{45} \mathrm{~s}^{-1}$, which suggests that CNS is being photoionized by a B1 star (Panagia 1973).

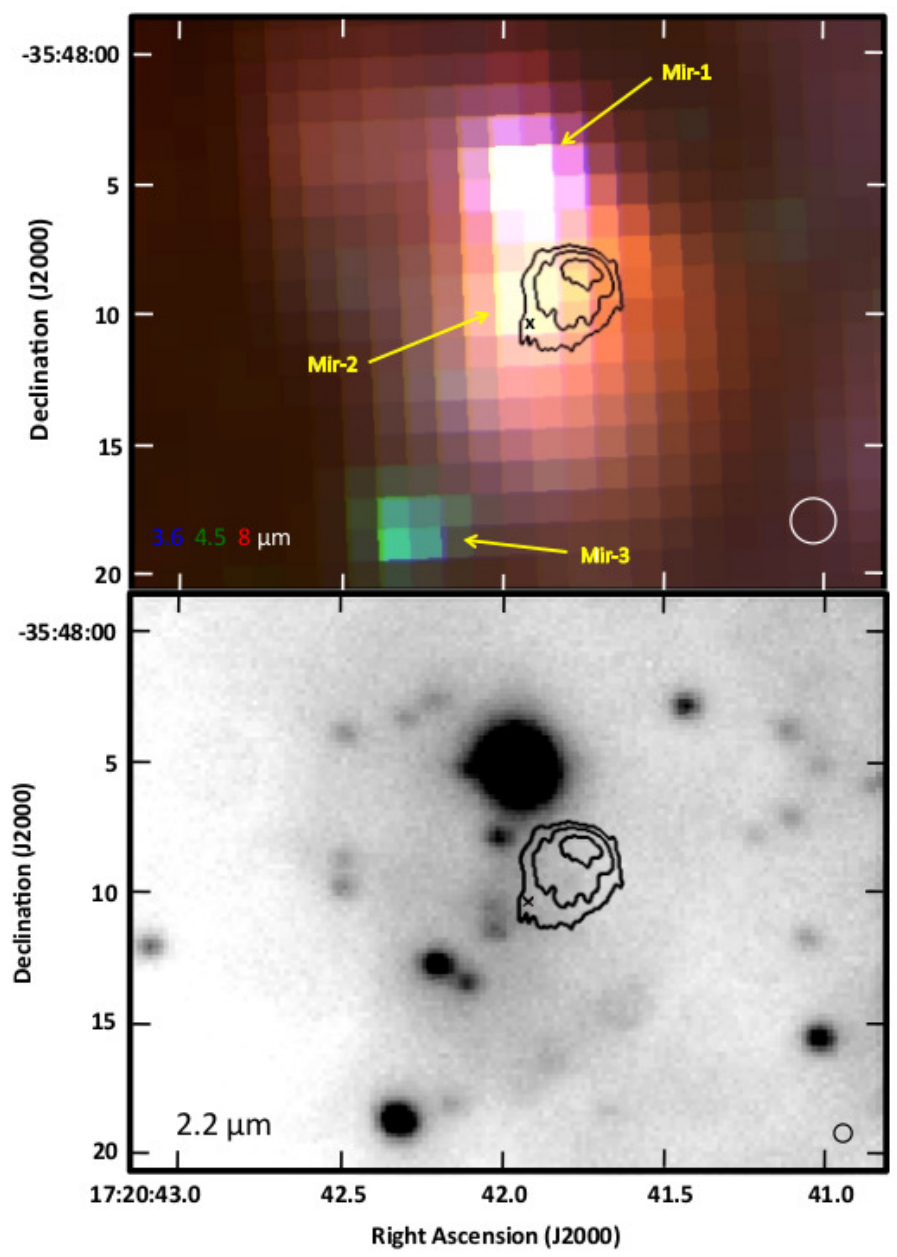

Fig. 7. Top: composite color image of the infrared nebula coincident with VLA172041.75-3548082 (CNN) in the IRAC bands centered at 3.6 (blue), 4.5 (green), and $8 \mu \mathrm{m}$ (red). Superposed in black are the 6.0 GHz contours (levels are the same as Fig. 2) with the small cross marking the position of the compact source. The three brightest mid-IR unresolved sources are labeled (Table 2). The white circle represents the mean resolution of the IRAC images. Bottom: same as 7-Top, showing a higher-resolution $\left(0.7^{\prime \prime}\right) 2.2$ um image taken with the DuPont telescope at Las Campanas Observatory (Tapia et al., in prep.).

\subsubsection{Double nebula source $($ DNS $)=$ VLA J172044.4-354917}

Most intriguing is the double nebula source (DNS), radio source VLA J172044.47-3549017, located almost at the center of the radio H II region D (e.g., the $1.6 \mathrm{GHz}$ map by Brooks \& Whiteoak 2001). This source is surrounded by three radio compact sources (VLA 13, VLA 14, and VLA 15) with IR counterparts, see Fig. 10. Their IR counterparts indicate that they are massive stars with spectral types from late to early-B.

The nature of the double radio source is unknown. As can be seen in Figs. 1 and 10, there is a lot of diffuse emission in region D. Thus, we speculate that DNS is gas ionized by the above mentioned three massive stars.

To test this hypothesis we analyzed additional VLA archive data. The observations were made on 2013 July 25 in L (1 to $2 \mathrm{GHz}$ ) band under project $13 \mathrm{~A}-448$. The array was in the $\mathrm{C}$ configuration. The data were calibrated following the standard CASA procedures. To obtain the best angular resolution possible an image was made using the spectral window with the highest frequency, centered at $1.92 \mathrm{GHz}$ and with a bandwidth 

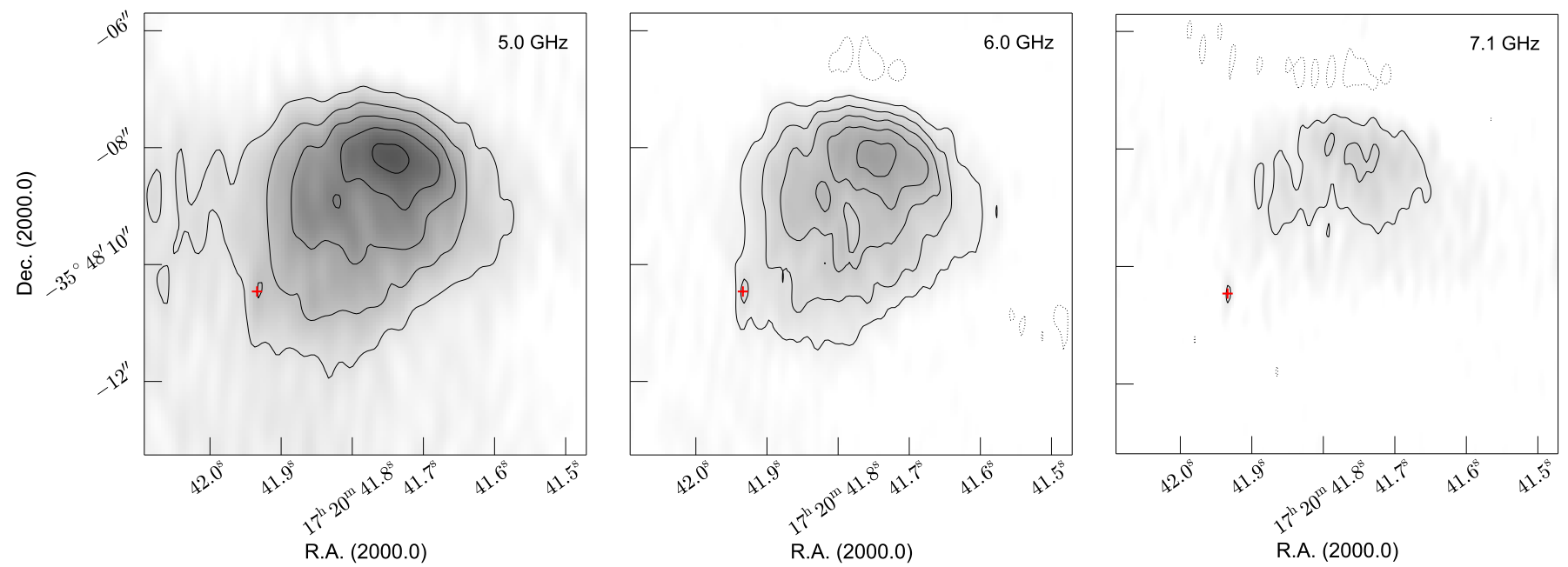

Fig. 8. Radio emission at the different frequencies from the cometary source close to region D. The noise levels around the source at each frequency are $25 \mu \mathrm{Jy}, 15 \mu \mathrm{Jy}$, and $19 \mu \mathrm{Jy}$ for the $5.0 \mathrm{GHz}, 6.0 \mathrm{GHz}$, and $7.1 \mathrm{GHz}$ maps, respectively. The contour levels are $-3,5,10,15,20$, and 25 times the noise level on each map. The red cross indicates the position of a source that is present in the three maps and may be related to Mir- 2 .

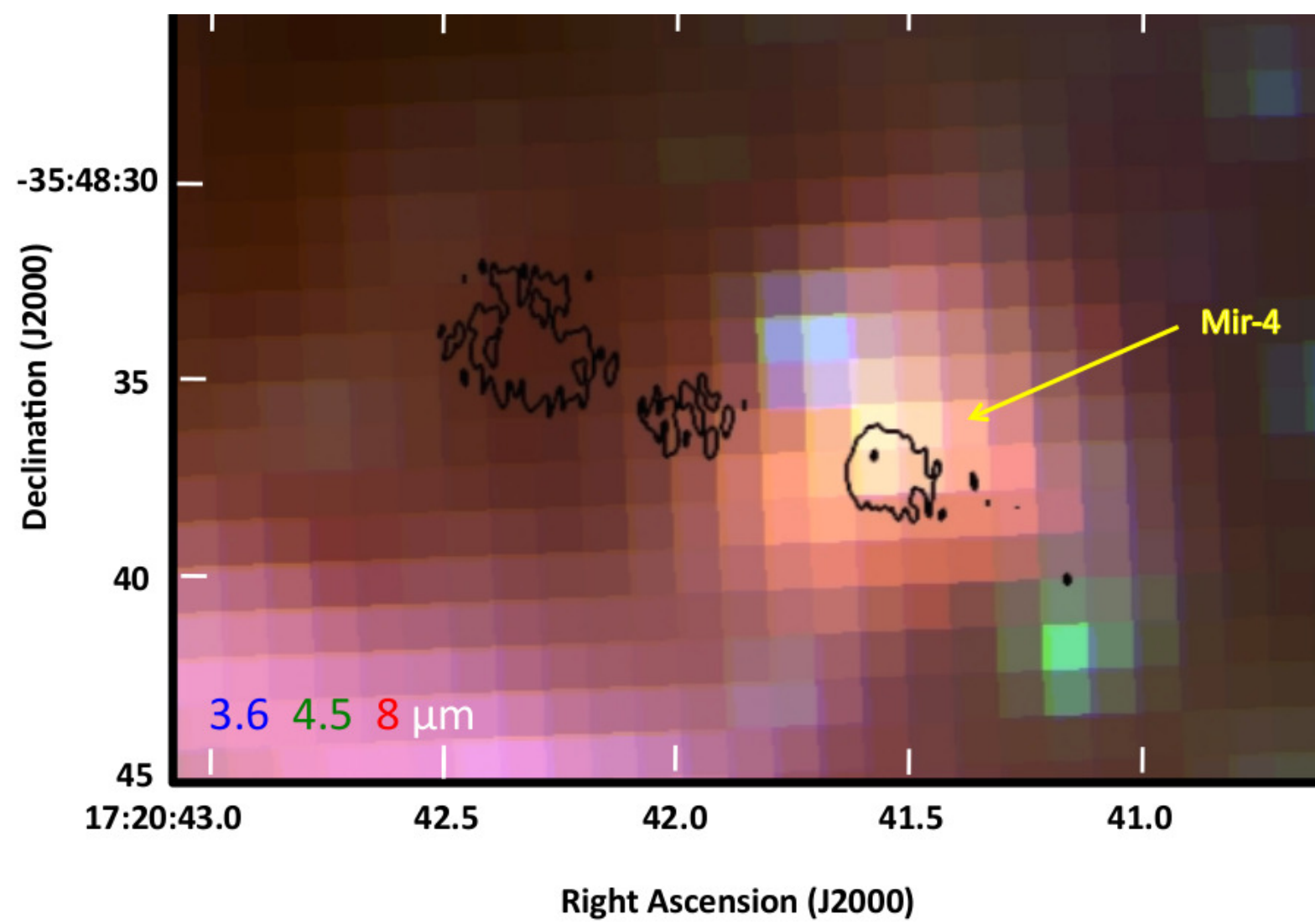

Fig. 9. Composite infrared image around VLA J172041.59-354837.3 (CNS). Contours correspond to the radio image at $6.0 \mathrm{GHz}$, and contour levels are the same as Fig. 2. The yellow arrow indicate the positions of Mir-4 source.

of $128 \mathrm{MHz}$. We additionally used superuniform weighting in the CLEAN task of CASA. The image was also corrected for the response of the primary beam and is shown in blue contours in Fig. 10. This image indicates that source D has a shell-like morphology and that the double radio source coincides with the brightest, eastern edge of the shell. Then, the double source may not be a true independent source but simply the brightest part of a larger structure.

\subsection{The central source in the $\mathrm{C}-\mathrm{H} I \mathrm{l}$ region $\mathrm{E}$}

This compact radio source was discovered by Carral et al. (2002) close to the center of the $\mathrm{C}-\mathrm{H}$ in region $\mathrm{E}$ (see Fig. 11) and it is our source No. 42 (Tables A.1 and 1). It is coincident with IR source 161 of Tapia et al. (1996), and was undetected in Feigelson et al. (2009) X-ray survey. Carral et al. (2002) measured a spectral index of $\alpha=1.0 \pm 0.7$ which is compatible with an ionized stellar 


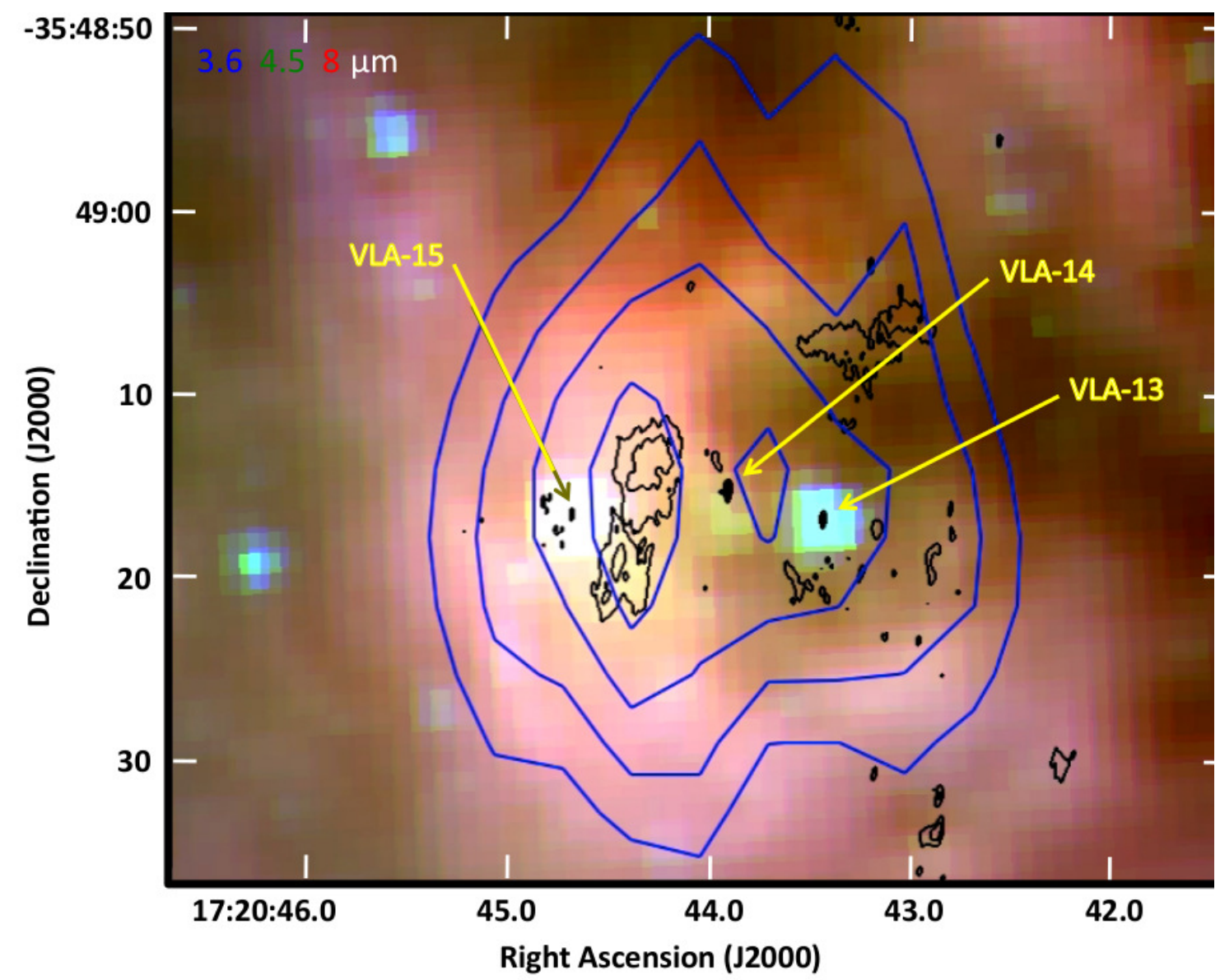

Fig. 10. Composite infrared image around VLA J1720444.4-354917 (DNS). Black contours correspond to the radio image at $6.0 \mathrm{GHz}$, and contour levels are the same as Fig. 2. Blue contours correspond to the image $1.92 \mathrm{GHz}$, and the contour levels are $0.32,0.37,0.41 \mathrm{and} 0.46 \mathrm{Jy} / \mathrm{beam}$. The beam size of this image is $26^{\prime \prime} 3 \times 66^{\prime \prime} 4 ; \mathrm{PA}=-179^{\circ}$.

envelope and, thus, they interpreted this source as the ionizing star of source E. The IR photometry here reported confirms that this is a Class I YSO, with $A_{V}>55$ and $L_{\mathrm{IR}}>10 L_{\odot}$. Lacking mid- and far-IR fluxes, we can neither rule out nor confirm that this star is the ionizing star of the $\mathrm{C}-\mathrm{H}_{\text {II }}$ region, though the spatial coincidence makes it very probable.

The measured spectral index in our maps is $\alpha=1.1 \pm 0.3$ which is consistent with the previous results and the thermal nature of this source is corroborated. Using the estimated spectral index to extrapolate our measured flux to $8.4 \mathrm{GHz}$, we obtain a flux density of $1.2 \pm 0.3 \mathrm{mJy}$, which is in good agreement with the flux density measured by Carral et al. (2002). This nonvariability also supports the thermal nature of the radio source.

\subsection{Long term variability}

Most of the previous radio observations on NGC 6334 were focused on regions $\mathrm{E}, \mathrm{F}$, and $\mathrm{I}(\mathrm{N})$, as they have shown the most recent star formation on this molecular cloud. Furthermore, they reached noise levels that were much poorer than those of the final images reported on this paper. Still, we may use these observations to roughly measure variability on some sources. In the VLA archive we found a VLA observation, in A configuration, obtained on 11 August 1995 at $8.4 \mathrm{GHz}$, that is part of project AM495. These observations were reported in Carral et al. (2002). The pointing center was $\mathrm{RA}=17^{\mathrm{h}} 20^{\mathrm{m}} 53.40$; Dec. $=-35^{\circ} 46^{\prime} 25^{\prime \prime} .0$ (i.e., roughly equidistant from regions $\mathrm{E}, \mathrm{F}$, and $\mathrm{I}(\mathrm{N}))$. The data were edited and calibrated in a standard way.
Images were produced with a pixel size of $0^{\prime \prime} .06$ and, as it was done by Carral et al. (2002), removing the short spacings below $100 \mathrm{k} \lambda$ (to filter out structures larger than $2^{\prime \prime}$ ). The final beam size of this image was $00^{\prime \prime} 48 \times 0^{\prime \prime} .19 ; \mathrm{PA}=0^{\circ} .7$. We look at the position of sources in Table A.1 and that are inside the imaged area. In this case, we used a threshold of three times the noise level to consider a detection. Five sources were detected using this restriction and their fluxes from this image are reported in Table 3. Additionally, using the calculated spectral index, we extrapolate our measured fluxes at $6.0 \mathrm{GHz}$ and determine their fluxes at $8.4 \mathrm{GHz}$, these are also listed on Table 3. Most of the sources are in good agreement with their expected flux in 2011, which suggests that these sources are not strongly variables. The only exception is source VLA J172058.14-354934.6 which shows a decrease in its flux from 1995 to 2011 by a factor of $2.4 \pm 0.3$.

On the other hand, we notice that due to the smaller imaged area and larger noise, most of the sources are not expected to be detected because they fall outside the primary beam or their flux will be below three times the noise level. However, using the extrapolated flux in epoch 2011 and assuming non-variability, three sources should be detected above this threshold in the $1995 \mathrm{im}-$ age. Interestingly, they are not. In Table 3 we list these sources, an upper limit of three times the noise level (with the noise level of the area as the flux error) and its predicted flux at $8.4 \mathrm{GHz}$ for the epoch 2011. Clearly sources VLA J172052.02-354938.0 and VLA J172053.65-354548.4 are strong variables ( $\gtrsim 100 \%)$ on scales of years. We could not support a variability for source VLA J172057.98-354431.6, since its non-detection may be due 


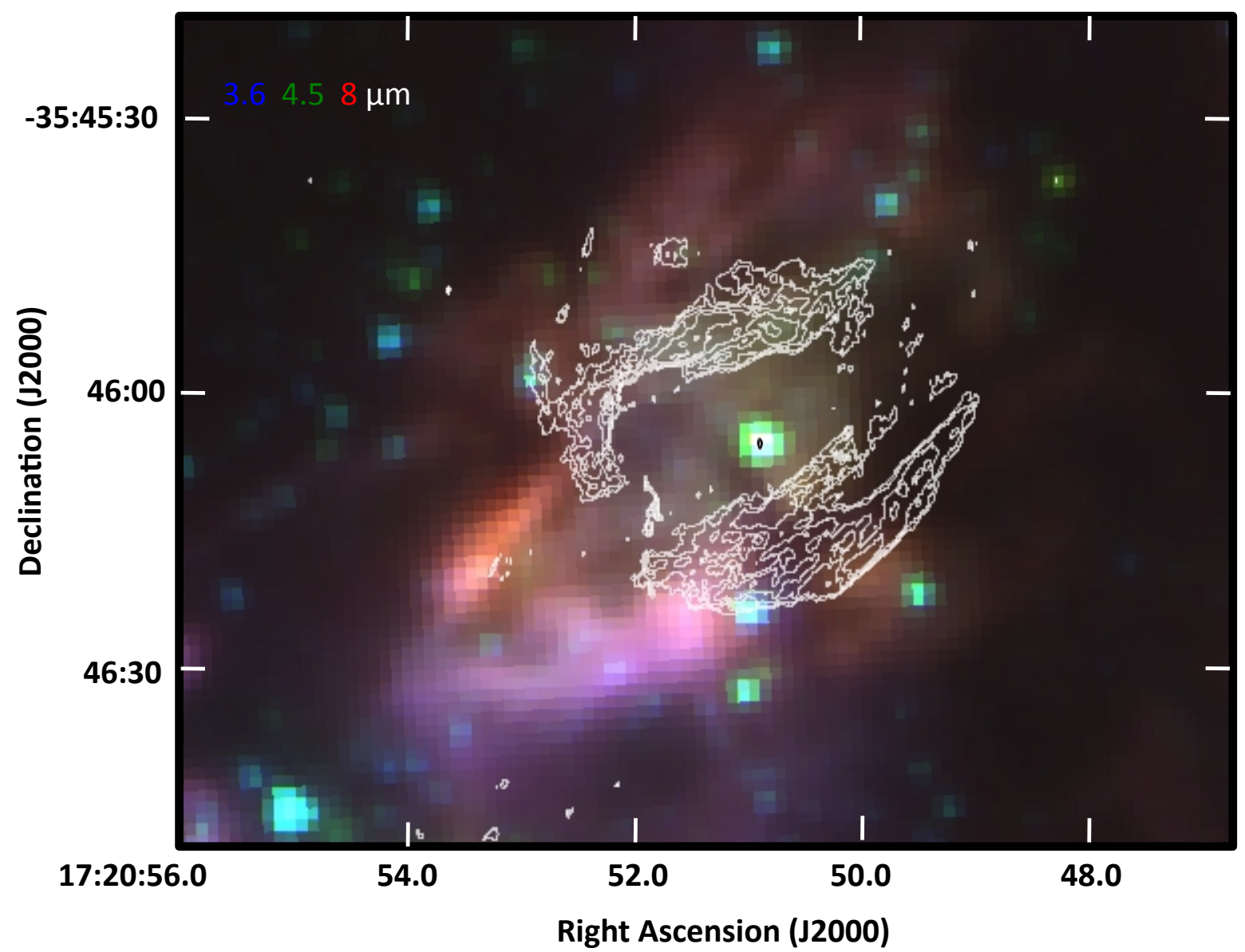

Fig. 11. Composite infrared image around VLA J172050.91-354605.0 (the source near the center of region E). Contours correspond to the radio image at $6.0 \mathrm{GHz}$, and contour levels are the same as Fig. 2.

only to fluctuation in the flux caused by the noise of the observations.

We also searched for possible additional new sources by using BLOBCAT and with the parameters used for the $6.0 \mathrm{GHz}$ map. We did not find any additional new sources.

The three strongly variable sources reported here may be good examples of magnetically active YSOs. With future deep observations we could study variability from the weakest radio sources.

\subsection{Other sources}

We could not determine the spectral classification for most of the remaining radio sources. However, the infrared emission of two sources, 24 and 50 in Table A.1, indicate that they are early B stars. Their spectral index has large errors but suggests a flat spectrum, so they are most probably thermal emitters. The radio emission in these cases may originate in the winds of the massive stars.

It is hard to speculate on the radio emission of the remaining sources, because of the scarce information. Future multiwavelength and multi-epoch observations with better sensitivity may help to reveal their nature.

\section{Conclusions}

We have presented a deep radio observation $\left(\sigma \sim 50 \mu \mathrm{Jy} \mathrm{bm}^{-1}\right)$ with high angular resolution $\left(0^{\prime \prime} .2\right)$ of the NE of the NGC 6334
Table 3. Comparison from radio sources of epoch 1995 and 2011.

\begin{tabular}{crr}
\hline \hline VLA name & $\begin{array}{r}S_{v}(1995) \\
(\mu \mathrm{Jy})\end{array}$ & $\begin{array}{r}S_{v}(2011) \\
(\mu \mathrm{Jy})\end{array}$ \\
\hline $\mathrm{J} 172103.47-354618.8$ & $406 \pm 59$ & $453 \pm 52$ \\
$\mathrm{~J} 172052.02-354938.0$ & $<408 \pm 136$ & $1038 \pm 93$ \\
$\mathrm{~J} 172053.65-354548.4$ & $<171 \pm 57$ & $338 \pm 62$ \\
$\mathrm{~J} 172057.98-354431.6$ & $<216 \pm 72$ & $220 \pm 24$ \\
$\mathrm{~J} 172050.91-354605.0$ & $1380 \pm 110$ & $1188 \pm 290$ \\
$\mathrm{~J} 172055.19-354503.8$ & $401 \pm 107$ & $365 \pm 23$ \\
$\mathrm{~J} 172058.14-354934.6$ & $1240 \pm 110$ & $510 \pm 52$ \\
$\mathrm{~J} 172054.62-354508.5$ & $290 \pm 60$ & $192 \pm 25$ \\
\hline
\end{tabular}

Notes. Upper-limits to fluxes are obtained as three times the noise level, that are at the same time used as the error for these upper-limits.

complex (covering the regions D, E, F, I(N), and part of the region C) searching for compact radio sources. We also searched for infrared counterparts of detected compact radio sources and characterized them. The results and conclusions from our analysis are listed below.

- A total of 83 radio compact sources in the NE of NGC 6334 are detected, 15 of them are located inside the region D. Most of these sources are new detections and only around 10 of 
them were previously reported and are located in regions $\mathrm{E}$ $\mathrm{I}$, and $\mathrm{I}(\mathrm{N})$.

- The stellar nature of 27 of the 83 compact radio sources is confirmed by the properties of their infrared emission.

- We computed the spectral index of the sources in order to speculate about the nature of their radio emission. In region $\mathrm{D}$ the values tend to be negative, suggesting nonthermal emission. Most of these sources are likely magnetically active low mass YSOs as in the Orion core. However, the IR emission of three of them suggest that they are early B stars and their radio emission may originate in strong shocks of wind collision regions.

- Two interesting cometary radio sources, CNN and CNS, were detected close to region $\mathrm{D}$ and are here reported for the first time. They are spatially coincident with more extended mid-IR nebulosities of similar shape. We suggest that they are $\mathrm{H}_{\text {II }}$ regions (traced by the radio emission) surrounded by dusty envelopes (traced by the mid-IR). Interestingly we found three stars (Mir-1, Mir-2, and Mir-3) with spectral types earlier than A, which could be the sources of the ionizing photons of CNN. On the other hand, the possible ionizing source of CNS is Mir-4.

- Through the inspection of an additional $1.92 \mathrm{GHz}$ image, we suggest that the double source VLA J1720444.4-354917 (DNS) is part of the diffuse ionized gas from the region D.

- Our observations support the thermal nature of source VLA $\mathrm{J} 172050.91-354605.0$, which is located near the center of the radio $\mathrm{H}_{\text {II }}$ region $\mathrm{E}$.

- By comparing with an observation obtained in 1995, we analyzed the variability in flux of eight sources. Three of them show strong variability suggesting that they are magnetically active low mass stars.

Our analysis has provided clues as to the nature of several of the detected compact radio sources. However, future observations are necessary to better establish the nature of most of these sources. Ideally, these observation should be multi-wavelength and multi-epoch.

Acknowledgements. S.-N.X.M. acknowledges IMPRS for a Ph.D. research scholarship. L.F.R., M. T. and L.L. acknowledge the financial support of DGAPA, UNAM, and CONACyT, México. M.T. and L.L. acknowledge support for this work through UNAM-PAPIIT grants IN104316, and IN112417. This paper makes use of archival data obtained with the Spitzer Space Telescope, which is operated by the Jet Propulsion Laboratory, California Institute of Technology (CIT) under National Aeronautics and Space Administration (NASA) contract 1407. This research has made use of the NASA/ IPAC Infrared Science Archive, which is operated by the Jet Propulsion Laboratory, California Institute of Technology, under contract with the National Aeronautics and Space Administration. This research has made use of the SIMBAD database, operated at CDS, Strasbourg, France.

\section{References}

Andre, P. 1996, in Radio Emission from the Stars and the Sun, ASP Conf. Ser., 93, 273

Anglada, G. 1996, in Radio Emission from the Stars and the Sun, ASP Conf. Ser., 93, 3

Anglada, G., Villuendas, E., Estalella, R., et al. 1998, AJ, 116, 2953

Bassani, L., De Rosa, A., Bazzano, A., et al. 2005, ApJ, 634, L21

Bertin, E., \& Arnouts, S. 1996, A\&AS, 117, 393

Briggs, D. S. 1995, BAAS, 27, 112.02

Brogan, C. L., Hunter, T. R., Cyganowski, C. J., et al. 2016, ApJ, 832, 187

Brooks, K. J., \& Whiteoak, J. B. 2001, MNRAS, 320, 465

Carral, P., Kurtz, S. E., Rodríguez, L. F., et al. 2002, AJ, 123, 2574

Contreras, M. E., Rodriguez, L. F., Gomez, Y., \& Velazquez, A. 1996, ApJ, 469, 329

Cutri, R. M., Skrutskie, M. F., van Dyk, S., et al. 2003, VizieR Online Data Catalog: II/246

Drilling, J. S., \& Landolt, A. U. 2000, Allen's Astrophysical Quantities, 381

Dzib, S. A., Rodríguez, L. F., Araudo, A. T., \& Loinard, L. 2013, Rev. Mex. Astron. Astrofis., 49, 345

Feigelson, E. D., Martin, A. L., McNeill, C. J., Broos, P. S., \& Garmire, G. P. 2009, AJ, 138, 227

Flaherty, K. M., Pipher, J. L., Megeath, S. T., et al. 2007, ApJ, 663, 1069

Forbrich, J., Rivilla, V. M., Menten, K. M., et al. 2016, ApJ, 822, 93

Hales, C. A., Murphy, T. Curran, J. R., et al. 2012, MNRAS, 425, 979

Holwerda, B. W. 2005, ArXiv e-prints [arXiv:astro-ph/0512139]

Hunter, T. R., Brogan, C. L., Megeath, S. T., et al. 2006, ApJ, 649, 888

Hunter, T. R., Brogan, C. L., Cyganowski, C. J., \& Young, K. H. 2014, ApJ, 788, 187

Koornneef, J. 1983, A\&A, 128, 84

Kounkel, M., Hartmann, L., Loinard, L., et al. 2017, ApJ, 834, 142

Kurtz, S. 2005, in Massive Star Birth: A Crossroads of Astrophysics, IAU Symp. Proc., 227, 111

López-Chico, T., \& Salas, L. 2007, Rev. Mex. Astron. Astrofis., 43, 155

Masqué, J. M., Rodríguez, L. F., Trinidad, M. A., et al. 2017, ApJ, 836, 96

Menten, K. M., Reid, M. J., Forbrich, J., \& Brunthaler, A. 2007, A\&A, 474, 515

Moran, J. M., Rodriguez, L. F., Greene, B., \& Backer, D. C. 1990, ApJ, 348, 147 O'dell, C. R., Wen, Z., \& Hu, X. 1993, ApJ, 410, 696

Ortiz-León, G. N., Loinard, L., Rodríguez, L. F., Mioduszewski, A. J., \& Dzib, S. A. 2011, ApJ, 737, 30

Persi, P., \& Tapia, M. 2008, in Handbook of Star Forming Regions, Volume II, 5,456

Rau, U., \& Cornwell, T. J. 2011, A\&A, 532, A71

Reid, M. J., Menten, K. M., Brunthaler, A., et al. 2014, ApJ, 783, 130

Rieke, G. H., \& Lebofsky, M. J. 1985, ApJ, 288, 618

Rodriguez, L. F., Canto, J., \& Moran, J. M. 1982, ApJ, 255, 103

Rodríguez, L. F., Zapata, L. A., \& Ho, P. T. P. 2007, ApJ, 654, L143

Rodríguez, L. F., González, R. F., Montes, G., et al. 2012, ApJ, 755, 152

Sandell, G. 2000, A\&A, 358, 242

Straw, S. M., Hyland, A. R., \& McGregor, P. J. 1989, ApJS, 69, 99

Tapia, M. 1981, MNRAS, 197, 949

Tapia, M., Persi, P., \& Roth, M. 1996, A\&A, 316, 102

Tapia, M., Persi, P., Roth, M., et al. 2014, MNRAS, 437, 606

Willis, S., Marengo, M., Allen, L., et al. 2013, ApJ, 778, 96

Ybarra, J. E., Tapia, M., Román-Zúñiga, C. G., \& Lada, E. A. 2014, ApJ, 794, L25

Zapata, L. A., Rodríguez, L. F., Kurtz, S. E., \& O’Dell, C. R. 2004, AJ, 127, 2252 


\section{Appendix A: Additional table}

Table A.1. Radio properties of compact sources in NGC 6334.

\begin{tabular}{|c|c|c|c|c|c|c|c|}
\hline $\begin{array}{l}\text { ID } \\
\#\end{array}$ & VLA Name & $\begin{array}{l}\text { Other } \\
\text { Name }\end{array}$ & $\begin{array}{l}S_{v}(6 \mathrm{GHz}) \\
(\mu \mathrm{Jy})\end{array}$ & $\begin{array}{l}S_{v}(5 \mathrm{GHz}) \\
(\mu \mathrm{Jy})\end{array}$ & $\begin{array}{l}S_{v}(7 \mathrm{GHz}) \\
(\mu \mathrm{Jy})\end{array}$ & $\alpha$ & $S / N$ \\
\hline \multicolumn{8}{|c|}{ Sources inside region D } \\
\hline 1 & $\mathrm{~J} 172038.21-354846.8$ & $\ldots$ & $372 \pm 24$ & $421 \pm 27$ & $305 \pm 26$ & $-0.9 \pm 0.3$ & 28.7 \\
\hline 2 & J172038.43-354838.9 & $\ldots$ & $118 \pm 14$ & $141 \pm 17$ & $81 \pm 19$ & $-1.6 \pm 0.7$ & 9.4 \\
\hline 3 & J172039.07-354912.3 & $\ldots$ & $127 \pm 15$ & $125 \pm 17$ & $168 \pm 23$ & $0.9 \pm 0.6$ & 9.6 \\
\hline 4 & J172039.61-354935.7 & $\ldots$ & $80 \pm 16$ & $89 \pm 18$ & $<66 \pm 22$ & $<-0.9 \pm 1.1$ & 5.2 \\
\hline 5 & J172039.79-354906.7 & $\ldots$ & $144 \pm 17$ & $176 \pm 20$ & $116 \pm 21$ & $-1.2 \pm 0.6$ & 9.6 \\
\hline 6 & $\mathrm{~J} 172040.09-354852.7$ & $\ldots$ & $63 \pm 13$ & $70 \pm 17$ & $<63 \pm 21$ & $<-0.3 \pm 1.2$ & 5.1 \\
\hline 7 & $\mathrm{~J} 172040.19-354845.2$ & $\ldots$ & $76 \pm 15$ & $85 \pm 19$ & $98 \pm 11$ & $0.4 \pm 0.7$ & 5.7 \\
\hline 8 & $\mathrm{~J} 172040.40-354943.3$ & 2MASS J17204040-3549438 & $168 \pm 17$ & $143 \pm 18$ & $223 \pm 26$ & $1.3 \pm 0.5$ & 11.8 \\
\hline 9 & J172040.89-354856.4 & $\cdots$ & $81 \pm 13$ & $84 \pm 17$ & $75 \pm 17$ & $-0.3 \pm 0.9$ & 6.3 \\
\hline 10 & J172041.26-354941.7 & $\ldots$ & $77 \pm 15$ & $95 \pm 19$ & $<66 \pm 22$ & $<-1.0 \pm 1.1$ & 5.5 \\
\hline 11 & J172042.61-354854.9 & 2MASS J17204261-3548550 & $210 \pm 24$ & $185 \pm 28$ & $239 \pm 22$ & $0.7 \pm 0.5$ & 9.7 \\
\hline 12 & $\mathrm{~J} 172043.23-354902.2$ & -. & $236 \pm 28$ & $269 \pm 30$ & $189 \pm 20$ & $-1.0 \pm 0.4$ & 9.6 \\
\hline 13 & J172043.46-354917.0 & 2MASS J17204345-3549172 & $271 \pm 33$ & $266 \pm 44$ & $212 \pm 23$ & $-0.6 \pm 0.6$ & 9.3 \\
\hline 14 & J172043.92-354915.3 & $\ldots$ & $568 \pm 43$ & $612 \pm 50$ & $522 \pm 34$ & $-0.5 \pm 0.3$ & 18.4 \\
\hline 15 & J172044.66-354916.7 & 2MASS J17204466-3549168 & $129 \pm 12$ & $120 \pm 13$ & $126 \pm 13$ & $0.1 \pm 0.4$ & 5.1 \\
\hline \multicolumn{8}{|c|}{ Remaining Sources } \\
\hline 16 & J172028.97-354702.4 & $\cdots$ & $109 \pm 22$ & $<105 \pm 35$ & $\cdots$ & $\cdots$ & 5.1 \\
\hline 17 & J172029.78-355055.5 & $\ldots$ & $305 \pm 60$ & $210 \pm 42$ & $\ldots$ & $\ldots$ & 5.7 \\
\hline 18 & J172030.69-354741.1 & $\cdots$ & $95 \pm 19$ & $73 \pm 19$ & $\cdots$ & $\ldots$ & 5.6 \\
\hline 19 & $\mathrm{~J} 172031.18-355048.3$ & $\cdots$ & $232 \pm 48$ & $116 \pm 34$ & $\cdots$ & $\cdots$ & 5.0 \\
\hline 20 & $\mathrm{~J} 172033.41-354542.5$ & $\ldots$ & $85 \pm 17$ & $72 \pm 18$ & $\ldots$ & $\ldots$ & 5.5 \\
\hline 21 & $\mathrm{~J} 172034.23-355051.6$ & $\ldots$ & $793 \pm 57$ & $772 \pm 52$ & $\ldots$ & $\cdots$ & 20.8 \\
\hline 22 & J172034.55-354645.9 & $\cdots$ & $70 \pm 14$ & $64 \pm 17$ & $\cdots$ & $\cdots$ & 5.0 \\
\hline 23 & J172034.75-354543.9 & $\cdots$ & $70 \pm 14$ & $54 \pm 17$ & $\cdots$ & $\cdots$ & 5.3 \\
\hline 24 & J172035.44-354336.9 & $\cdots$ & $114 \pm 23$ & $91 \pm 22$ & $\cdots$ & $\cdots$ & 5.1 \\
\hline 25 & J172039.12-354510.5 & 2MASS J17203909-3545108 & $63 \pm 12$ & $71 \pm 15$ & $75 \pm 20$ & $0.2 \pm 1.0$ & 5.7 \\
\hline 26 & J172039.35-354656.3 & $\cdots$ & $49 \pm 10$ & $66 \pm 15$ & $<51 \pm 17$ & $<-0.7 \pm 1.2$ & 5.0 \\
\hline 27 & $\mathrm{~J} 172039.67-354632.5$ & $\ldots$ & $90 \pm 10$ & $98 \pm 13$ & $77 \pm 15$ & $-0.7 \pm 0.7$ & 9.7 \\
\hline 28 & $\mathrm{~J} 172039.77-354309.2$ & $\cdots$ & $106 \pm 21$ & $80 \pm 22$ & $\cdots$ & $\cdots$ & 5.3 \\
\hline 29 & $\mathrm{~J} 172040.21-355059.3$ & $\ldots$ & $131 \pm 21$ & $103 \pm 21$ & $\ldots$ & $\ldots$ & 6.5 \\
\hline 30 & $\mathrm{~J} 172040.84-354639.0$ & $\cdots$ & $68 \pm 10$ & $84 \pm 13$ & $53 \pm 13$ & $-1.3 \pm 0.8$ & 7.4 \\
\hline 31 & $\mathrm{~J} 172040.87-355125.4$ & $\cdots$ & $130 \pm 26$ & $106 \pm 24$ & $\ldots$ & $\cdots$ & 5.1 \\
\hline 32 & J172043.76-355039.9 & $\ldots$ & $877 \pm 50$ & $976 \pm 56$ & $\ldots$ & $\ldots$ & 53.8 \\
\hline
\end{tabular}

Notes. Other names are obtained from the following catalogs: $2 \mathrm{MASS}=$ Cutri et al. (2003); CXOU = Feigelson et al. (2009); [HBM2006b] $=$ Hunter et al. (2006); CM = Brogan et al. (2016); SSTU = Willis et al. (2013); [S2000e] = Sandell (2000); and [HBC2014] = Hunter et al. (2014). The $\mathrm{S} / \mathrm{Ns}$ are obtained from the $6.0 \mathrm{GHz}$ map. Upper-limits to fluxes are obtained as three times the noise level in the image, that are at the same time used as the error for these upper-limits. 
Table A.1. continued.

\begin{tabular}{|c|c|c|c|c|c|c|c|}
\hline $\begin{array}{l}\text { ID } \\
\#\end{array}$ & VLA Name & $\begin{array}{l}\text { Other } \\
\text { Name }\end{array}$ & $\begin{array}{l}S_{v}(6 \mathrm{GHz}) \\
(\mu \mathrm{Jy})\end{array}$ & $\begin{array}{l}S_{v}(5 \mathrm{GHz}) \\
(\mu \mathrm{Jy})\end{array}$ & $\begin{array}{l}S_{v}(7 \mathrm{GHz}) \\
(\mu \mathrm{Jy})\end{array}$ & $\alpha$ & $S / N$ \\
\hline 33 & $\mathrm{~J} 172044.36-355027.2$ & $\ldots$ & $76 \pm 15$ & $77 \pm 19$ & $\ldots$ & $\ldots$ & 5.2 \\
\hline 34 & $\mathrm{~J} 172045.47-354624.7$ & $\ldots$ & $41 \pm 08$ & $38 \pm 11$ & $41 \pm 11$ & $0.2 \pm 1.1$ & 5.3 \\
\hline 35 & J172046.42-354537.6 & $\ldots$ & $83 \pm 09$ & $73 \pm 12$ & $95 \pm 12$ & $0.8 \pm 0.6$ & 10.1 \\
\hline 36 & J172046.82-354426.1 & $\ldots$ & $53 \pm 10$ & $<60 \pm 20$ & $<48 \pm 16$ & $\ldots$ & 5.2 \\
\hline 37 & J172047.65-354447.4 & $\ldots$ & $52 \pm 10$ & $41 \pm 13$ & $59 \pm 14$ & $1.0 \pm 1.1$ & 5.3 \\
\hline 38 & J172048.27-355133.6 & $\ldots$ & $126 \pm 21$ & $109 \pm 21$ & $\ldots$ & $\ldots$ & 6.7 \\
\hline 39 & J172048.28-354536.6 & SSTU J172048.27-354537.0 & $80 \pm 11$ & $98 \pm 17$ & $55 \pm 13$ & $-1.7 \pm 0.8$ & 7.8 \\
\hline 40 & J172048.64-354336.8 & $\ldots$ & $62 \pm 13$ & $71 \pm 15$ & $<69 \pm 23$ & $<-0.1 \pm 1.2$ & 5.0 \\
\hline 41 & J172049.16-354640.4 & $\ldots$ & $41 \pm 08$ & $40 \pm 12$ & $40 \pm 12$ & $0.0 \pm 1.2$ & 5.4 \\
\hline 42 & $\mathrm{~J} 172050.91-354605.0$ & 2MASS J17205087-3546047 & $821 \pm 51$ & $612 \pm 64$ & $889 \pm 52$ & $1.1 \pm 0.3$ & 32.5 \\
\hline 43 & J172051.86-354704.5 & CM1 & $85 \pm 12$ & $72 \pm 13$ & $104 \pm 16$ & $1.1 \pm 0.7$ & 7.4 \\
\hline 44 & $\mathrm{~J} 172052.02-354938.0$ & CXOU 172052.0-354938 & $878 \pm 48$ & $834 \pm 47$ & $978 \pm 55$ & $0.5 \pm 0.2$ & 87.4 \\
\hline 45 & $\mathrm{~J} 172053.26-354305.7$ & [S2000e] SM6 & $90 \pm 15$ & $105 \pm 17$ & $\ldots$ & $\ldots$ & 6.4 \\
\hline 46 & $\mathrm{~J} 172053.38-354655.8$ & $\mathrm{CM} 2$ & $256 \pm 41$ & $266 \pm 75$ & $233 \pm 21$ & $-0.4 \pm 0.9$ & 6.6 \\
\hline 47 & $\mathrm{~J} 172053.42-354657.7$ & [HBM2006b] I-SMA1 & $466 \pm 70$ & $551 \pm 107$ & $608 \pm 131$ & $0.3 \pm 0.8$ & 7.1 \\
\hline 48 & $\mathrm{~J} 172053.65-354548.4$ & $\ldots$ & $258 \pm 16$ & $230 \pm 17$ & $307 \pm 19$ & $0.8 \pm 0.3$ & 31.2 \\
\hline 49 & J172054.06-354548.4 & SSTU J172053.96-354547.6 & $54 \pm 09$ & $50 \pm 10$ & $54 \pm 10$ & $0.2 \pm 0.8$ & 6.5 \\
\hline 50 & J172054.15-354513.7 & [HBC2014] $\mathrm{H}_{2} \mathrm{O}-\mathrm{C} 4$ & $98 \pm 10$ & $148 \pm 13$ & $75 \pm 11$ & $-1.1 \pm 0.4$ & 11.4 \\
\hline 51 & J172054.19-354554.0 & $\ldots$ & $61 \pm 09$ & $70 \pm 12$ & $73 \pm 10$ & $0.1 \pm 0.6$ & 7.5 \\
\hline 52 & $\mathrm{~J} 172054.26-355106.5$ & $\ldots$ & $80 \pm 16$ & $63 \pm 18$ & $\ldots$ & $\ldots$ & 5.3 \\
\hline 53 & J172054.34-354358.3 & SSTU J172054.33-354358.4 & $74 \pm 10$ & $71 \pm 14$ & $84 \pm 17$ & $0.5 \pm 0.8$ & 7.6 \\
\hline 54 & J172054.60-354516.9 & $\ldots$ & $45 \pm 08$ & $51 \pm 12$ & $60 \pm 11$ & $0.4 \pm 0.8$ & 5.7 \\
\hline 55 & $\mathrm{~J} 172054.62-354508.5$ & [HBM2006b] I(N)-SMA4 & $162 \pm 12$ & $152 \pm 14$ & $181 \pm 14$ & $0.5 \pm 0.3$ & 19.1 \\
\hline 56 & J172054.87-354536.5 & [HBC2014] VLA 2 & $86 \pm 10$ & $98 \pm 12$ & $64 \pm 11$ & $-1.2 \pm 0.6$ & 10.1 \\
\hline 57 & J172055.19-354503.8 & [HBM2006b] I(N)-SMA1b & $353 \pm 21$ & $347 \pm 22$ & $360 \pm 22$ & $0.1 \pm 0.3$ & 43.7 \\
\hline 58 & $\mathrm{~J} 172055.34-354758.7$ & $\ldots$ & $226 \pm 17$ & $212 \pm 21$ & $248 \pm 18$ & $0.5 \pm 0.4$ & 18.7 \\
\hline 59 & $\mathrm{~J} 172056.41-354921.0$ & $\ldots$ & $50 \pm 10$ & $<60 \pm 20$ & $<48 \pm 16$ & $\ldots$ & 5.1 \\
\hline 60 & $\mathrm{~J} 172056.47-354823.0$ & CXOU J172056.4-354823 & $55 \pm 10$ & $58 \pm 12$ & $62 \pm 11$ & $0.2 \pm 0.8$ & 5.5 \\
\hline 61 & $\mathrm{~J} 172056.96-354436.8$ & $\ldots$ & $46 \pm 09$ & $43 \pm 12$ & $66 \pm 13$ & $1.2 \pm 1.0$ & 5.2 \\
\hline 62 & J172057.62-354632.0 & $\ldots$ & $76 \pm 08$ & $105 \pm 13$ & $52 \pm 10$ & $-2.0 \pm 0.7$ & 10.4 \\
\hline 63 & J172057.98-354431.6 & $\ldots$ & $252 \pm 16$ & $272 \pm 19$ & $236 \pm 19$ & $-0.4 \pm 0.3$ & 27.6 \\
\hline 64 & J172058.14-354934.6 & $\ldots$ & $625 \pm 35$ & $689 \pm 39$ & $560 \pm 33$ & $-0.6 \pm 0.2$ & 62.9 \\
\hline
\end{tabular}


Table A.1. continued.

\begin{tabular}{llllllll}
\hline \hline ID & & Other & $\begin{array}{l}S_{v}(6 \mathrm{GHz}) \\
(\mu \mathrm{Jy})\end{array}$ & $\begin{array}{l}S_{v}(5 \mathrm{GHz}) \\
(\mu \mathrm{Jy})\end{array}$ & $\begin{array}{l}S_{v}(7 \mathrm{GHz}) \\
(\mu \mathrm{Jy})\end{array}$ & $\alpha$ & $S / N$ \\
$\#$ & VLA Name & Name & $55 \pm 10$ & $60 \pm 10$ & $<51 \pm 17$ & $<-0.5 \pm 1.1$ & 5.6 \\
\hline 65 & $\mathrm{~J} 172059.98-354947.5$ & $\ldots$ & $41 \pm 08$ & $45 \pm 11$ & $43 \pm 10$ & $-0.1 \pm 1.0$ & 5.4 \\
66 & $\mathrm{~J} 172100.08-354743.9$ & $\ldots$ & $157 \pm 11$ & $157 \pm 13$ & $153 \pm 13$ & $-0.1 \pm 0.3$ & 21.2 \\
67 & $\mathrm{~J} 172100.80-354626.5$ & $\ldots$ & $64 \pm 13$ & $65 \pm 14$ & $<60 \pm 20$ & $<-0.2 \pm 1.1$ & 5.1 \\
68 & $\mathrm{~J} 172101.31-355014.2$ & $\ldots$ & $41 \pm 08$ & $58 \pm 11$ & $<60 \pm 20$ & $<-1.9 \pm 1.1$ & 5.1 \\
69 & $\mathrm{~J} 172101.45-354534.0$ & $\ldots$ & $102 \pm 19$ & $96 \pm 20$ & $\ldots$ & $\ldots$ & 5.5 \\
70 & $\mathrm{~J} 172101.62-355106.2$ & $\ldots$ & $232 \pm 22$ & $221 \pm 22$ & $\ldots$ & $\ldots$ & 12.4 \\
71 & $\mathrm{~J} 172101.80-355106.4$ & $\ldots$ & $573 \pm 32$ & $639 \pm 36$ & $506 \pm 29$ & $-0.7 \pm 0.2$ & 73.8 \\
72 & $\mathrm{~J} 172103.47-354618.8$ & $\ldots$ & $44 \pm 09$ & $47 \pm 12$ & $38 \pm 12$ & $-0.6 \pm 1.2$ & 5.0 \\
73 & $\mathrm{~J} 172104.10-354540.5$ & $\ldots$ & $94 \pm 17$ & $70 \pm 12$ & $\ldots$ & $\ldots$ & 5.7 \\
74 & $\mathrm{~J} 172104.55-355033.1$ & $\ldots$ & $76 \pm 11$ & $57 \pm 13$ & $93 \pm 16$ & $1.4 \pm 0.8$ & 7.4 \\
75 & $\mathrm{~J} 172108.98-354714.4$ & $\ldots$ & $309 \pm 62$ & $208 \pm 43$ & $\ldots$ & $\ldots$ & 5.1 \\
76 & $\mathrm{~J} 172108.98-354153.8$ & $\ldots$ & $92 \pm 18$ & $83 \pm 22$ & $\ldots$ & $\ldots$ & 5.1 \\
77 & $\mathrm{~J} 172112.53-354921.4$ & $\ldots$ & $94 \pm 18$ & $70 \pm 18$ & $\ldots$ & $\ldots$ & 5.3 \\
78 & $\mathrm{~J} 172115.62-354548.6$ & $\ldots$ & $98 \pm 19$ & $75 \pm 18$ & $\ldots$ & $\ldots$ & 5.3 \\
79 & $\mathrm{~J} 172116.19-354554.1$ & $\ldots$ & $97 \pm 19$ & $78 \pm 19$ & $\ldots$ & $\ldots$ & 5.3 \\
80 & $\mathrm{~J} 172116.20-354627.6$ & $\ldots$ & $120 \pm 24$ & $85 \pm 22$ & $\ldots$ & $\ldots$ & 5.1 \\
81 & $\mathrm{~J} 172117.26-354823.5$ & $\ldots$ & $321 \pm 62$ & $207 \pm 43$ & $\ldots$ & $\ldots$ & 5.3 \\
82 & $\mathrm{~J} 172118.37-355032.2$ & $\ldots$ & $274 \pm 55$ & $<183 \pm 61$ & $\ldots$ & $\ldots$ & 5.2 \\
83 & $\mathrm{~J} 172118.54-354959.6$ & $\ldots$ & & & & & \\
\hline
\end{tabular}

